\title{
Statistical model of transient particle dispersion and deposition in vertical pipes
}

\author{
X.F. Loyseau, P.G. Verdin* \\ School of Water, Energy and Environment, Oil $\mathcal{G}$ Gas Engineering Centre \\ Cranfield University, Bedfordshire, MK43 OAL, United Kingdom
}

\begin{abstract}
Water droplets in vertical pipes have been investigated to assert the accuracy of a newly developed Lagrangian model for dispersion and deposition implemented in the Open source CFD code OpenFOAM. The transient evolution of the particles dispersion and concentration has been studied for the combined effects of Brownian motion and turbulent dispersion. A parametric study of mesh density has been performed and the influence of the isotropic representation of turbulence discussed. Simulated results have been compared to experimental data from the literature and to results generated with a commercial flow solver. A new model has also been developed to predict the evolution of the droplet concentration and deposition in pipes, based on a statistical description of the dispersion.
\end{abstract}

Keywords: multiphase flow, deposition, Lagrangian, droplets, dispersion, pipe

\section{Introduction}

Numerical methods able to predict the motion of droplets in a turbulent viscous flow are of major interest for numerous industrial applications, including hydrocarbon transportation in pipelines. Several simulation methods have been developed, tested and published, each method focusing in some specific aspects of these complex flows. A review of various techniques used to simulate such flows is provided by Loth (2000).

Dallali \& Armenio (2015) performed a two-way coupling Euler-Euler Large Eddy Simulation (LES) of particulate transport. They showed the effects of the near-wall structure and particle size on the concentration in a channel. They also showed how the particles affect and are affected by turbulence. Vreman (2007) conducted a four-way coupling when considering a two-phase flow in a vertical pipe and a Lagrangian approach. Further to turbulence effects, he studied the influence of various mass loadings on the flow. He proposed corrections for the discrepancies between simulation and experimental results. He also studied the turbulence attenuation in high mass loading flows, resulting in the disappearance of the high near-wall concentration, usually caused by turbophoresis. His simulations were based on a DNS (Direct Numerical Simulation) and included forces which are often omitted, such as the inter-particle drag. The same author (Vreman, 2015) further studied the turbulence attenuation by particles and investigated the impact of a rough wall on it, and gave a special attention to the feedback force (in particular in the determination of the contribution of the non-uniform part of this force). Marchioli \& Giusti (2003) performed a DNS study of a Lagrangian-based pipe flow deposition. This study allowed to correlate the sweeps and ejections events with the flux of particles going towards the wall or being driven away from it. They were able to predict accurately the deposition velocity for particles of particle relaxation times greater than 3 . They were thus able to show the acting forces in the deposition by studying the particle residence time near the wall. Picano et al. (2015) discussed the influence of the particle volume fraction on the mean flow quantities, based on a DNS perfromed with an immersed boundary method in a channel. More recently, De Marchis et al. (2016) conducted a DNS of Lagrangian particles in a geometrically rough domain to study particles reaction under the turbulence created by the geometry. They reported the PDF of the stream-wise and wall-normal particle velocities in the buffer layer; these are significantly different depending on the particle Stokes number. They allow to understand how each particle size moves into a different pattern.

\footnotetext{
*Corresponding author. Tel: +44 (0)1234 75011 ext.5214

Email address: p.verdin@cranfield.ac.uk (P.G. Verdin)
} 
DNS is the most accurate approach for studying the physics of the flow in pipes and other geometries. However, it is a time consuming technique, as detailed by Milici et al. (2014), who conducted a flow study in a rough channel. For most industrial applications, such detailed study is usually unnecessary. A compromise is often obtained with LES but this approach is also more time consuming than Reynolds Average Navier-Stokes Simulations (RANS). This has been demonstrated by Vijiapurapu \& Cui (2010) for simulations in a rough pipe where the authors claimed that a LES was three times longer than a Reynolds-stress based (RSM) simulation.

LES has not been used as the objective of the research presented here is to demonstrate that particle dispersion and deposition can be obtained through averaged methods, i.e. using RANS. Bearing in mind that some of the information related to turbulence is lost when using this approach, a simplified statistical model has been created to predict the deposition (mass rates and localized deposition sites) and concentration of droplets in a section of pipe.

The transport of particle can be studied from either an Eulerian or a Lagrangian point of view. While Eulerian approaches are widely used in multiphase flow applications due to their ability to deal with a large volume fraction of the particulate phase, a Lagrangian framework is preferred for accurate predictions, the forces being applied directly on each individual droplet (Gouesbet \& Berlemont, 1998; Zhang \& Chen, 2007; Doisneau et al., 2013). The access to particle statistics using a Lagrangian representation of the dispersed phase is also easier. In addition, it offers more control over the numerical implementation of the turbulence-induced particle dispersion and other localized effects. The turbulent dispersion plays a major role in the evaluation of the droplets dispersion and deposition, and is dependent on the particle relaxation time (Young \& Leeming, 1997). For these reasons, the Lagrangian approach has been preferred over the Eulerian approach.

The open source software OpenFOAM (Weller \& Tabor, 1998) has been selected as it offers the possibility to create, modify and implement models which might not be available in current commercial flow solvers. Droplet deposition results have been compared to those obtained with the industry standard CFD code FLUENT (2014) and with experimental data from the literature. In the following, a model is also proposed to predict the evolution of droplet concentration field and deposition rates. The objective is to establish a simplified model to predict the concentration pattern and deposition rates based on the statistical representation of the dispersion. This model has the advantage of requiring a simple RANS simulation to predict the average flow quantities and average turbulent intensities.

Information on fluctuations having a major impact on the particle motion which are lost when running a RANS simulation, can be modeled in the near-wall layer $\left(y^{+}<20\right)$ where the statistics of fluctuations are significantly different than those in the bulk flow (Herpin, 2009; Jin et al., 2015). However, an alternative method has been used in the present work, based on the Lagrangian Integral Timescale; this is described in details in Section 3.2. Such approach has been widely used and works reasonably well, see Lecrivain \& Hampel (2012) for details. Thus, by tuning the near wall lagrangian integral timescale, the effects resulting from a modification of the near-wall fluctuations PDF could be modeled.

Note that the dispersed phase concentration field can be reconstituted from a Lagrangian field, for instance using one of the methods reviewed by Marshall \& Sala (2013). This approach is mainly developed for low dispersed phase content as the flow conditions (in particular the turbulence) are modified by the particle fraction (Picano et al., 2015). However, the method presented here could be adapted to a higher dispersed phase content.

\section{Flow conditions}

Experiments were carried out by Liu \& Agarwal (1974) in a $1.016 \mathrm{~m}$ long vertical pipe with a $12.7 \mathrm{~mm}$ internal diameter. However, it has been established that a $1.016 \mathrm{~m}$ long pipe was not long enough to perform a complete and accurate study (Matida et al., 2000). The length of the pipe has therefore been increased to $5 \mathrm{~m}$. This has been found to be long enough to get accurate data for all droplet sizes. A mesh sensitivity analysis has been carried out to ensure that the evaluation of the flow fields (e.g. velocity, kinetic turbulent energy, turbulent dissipation rate) were not dependent on the grid density. Figure 1 shows the fully developed velocity profiles obtained for different regular meshes created with IcemCFD. Although the mesh comprising 464, 000 cells could have also been selected, the one comprising 760, 500 cells has been chosen as it was expected that it would produce a better evaluation of the droplets transport and deposition. This mesh is constituted of 100 cells of size $5 \mathrm{~cm}$ in the axial direction, 7, 605 cells in the radial direction ( $5 \mathrm{~cm}$ long at the center, $y^{+} \simeq 471$ ), and is refined next to the wall to ensure that the non-dimensional cell height is $y^{+} \simeq 1$. 


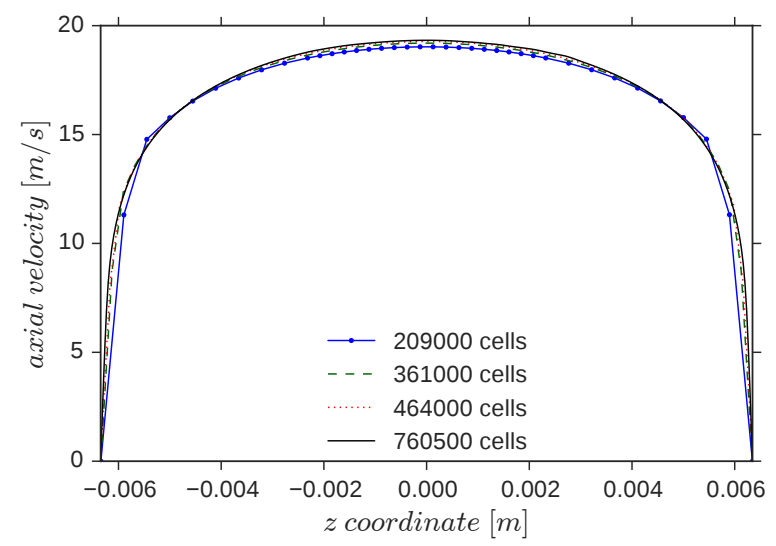

Figure 1: Mesh independence study

Simulations were performed with the flow conditions written in Table 1. Periodic boundary conditions were applied at the inlet and outlet sections of the pipe to ensure that the flow was fully developed inside the whole domain. The SIMPLE algorithm was run until convergence, with a convergence criterion of $10^{-6}$.

Table 1: Flow conditions for air in pipe

\begin{tabular}{ccccc}
\hline $\begin{array}{c}U_{G} \\
(\mathrm{~m} / \mathrm{s})\end{array}$ & $\begin{array}{c}\rho_{f} \\
\left(\mathrm{~kg} / \mathrm{m}^{3}\right)\end{array}$ & $\begin{array}{c}\mu_{f} \\
\left(\mathrm{~m}^{2} / \mathrm{s}\right)\end{array}$ & $\begin{array}{c}T \\
\left({ }^{\circ} \mathrm{C}\right)\end{array}$ & $\begin{array}{c}R e \\
(-)\end{array}$ \\
\hline 15.66 & 1.2 & $1.53410^{-5}$ & 21 & 15,000 \\
\hline
\end{tabular}

Several turbulence models implemented in OpenFOAM were tested. The Launder-Sharma k- $\varepsilon$ (Launder \& Sharma, 1974) and the shear stress transport (SST) k- $\omega$ (Menter, 1993) models were both able to predict a pressure drop in agreement with the Blasius correlation for smooth walls:

$$
\tau_{w}=0.3164 \cdot \mathrm{Re}^{-1 / 4} \cdot \rho_{f} U_{b u l k}^{2} / 8,
$$

which predicts a wall shear stress around $1.08 \mathrm{~Pa}$, leading to a pressure drop of $344 \mathrm{~Pa} / \mathrm{m}$.

Flow simulation results obtained with OpenFOAM have been compared to those from FLUENT, considering the same mesh and the k- $\omega$ SST turbulence model for both software. The SST formulation combines: i) the use of a $\mathrm{k}-\omega$ formulation in the inner parts of the boundary layer which makes this model directly usable in the viscous sublayer and can therefore be used as a Low-Re turbulence model, and ii) a switch to a k- $\varepsilon$ model in the bulk flow and therefore avoids sensitivity problems encountered by a k- $\omega$ formulation due to inlet turbulence properties (FLUENT, 2014). Figure 2(a) shows the corresponding mesh and Figure 2(b) the velocity profiles obtained with the open source and the commercial flow solvers. As can be seen, the velocity profiles are identical, this provides confidence in the flow settings and calculation with OpenFOAM. 


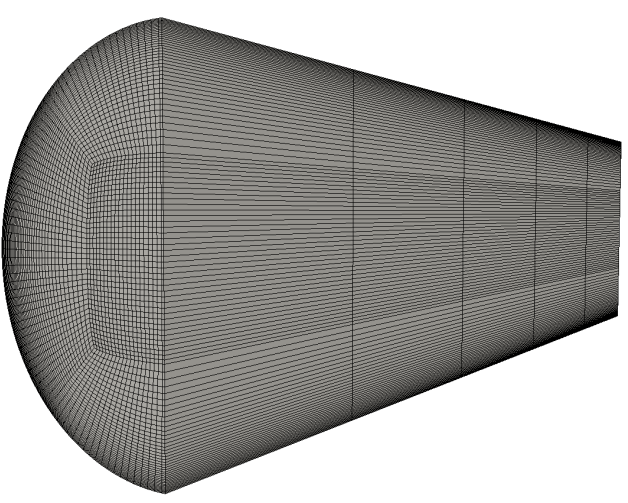

(a) Mesh detail

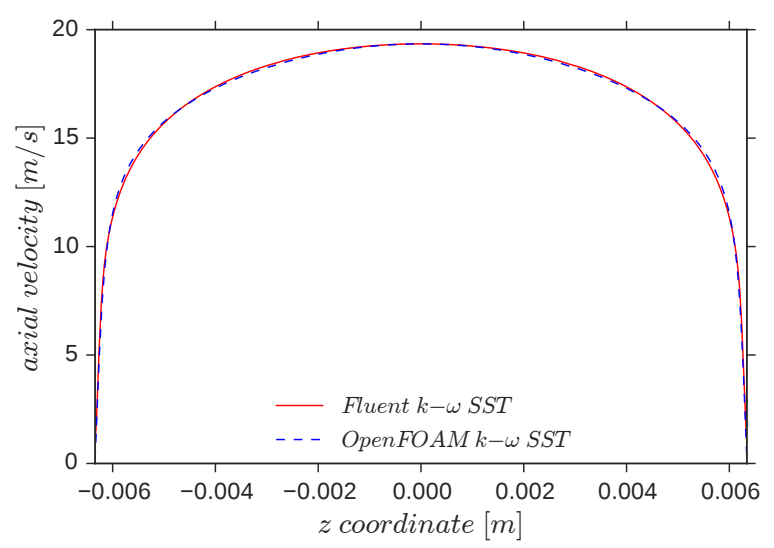

(b) Velocity profile comparison between OpenFOAM and Fluent

Figure 2: Mesh and velocity profiles obtained with OpenFOAM and Fluent

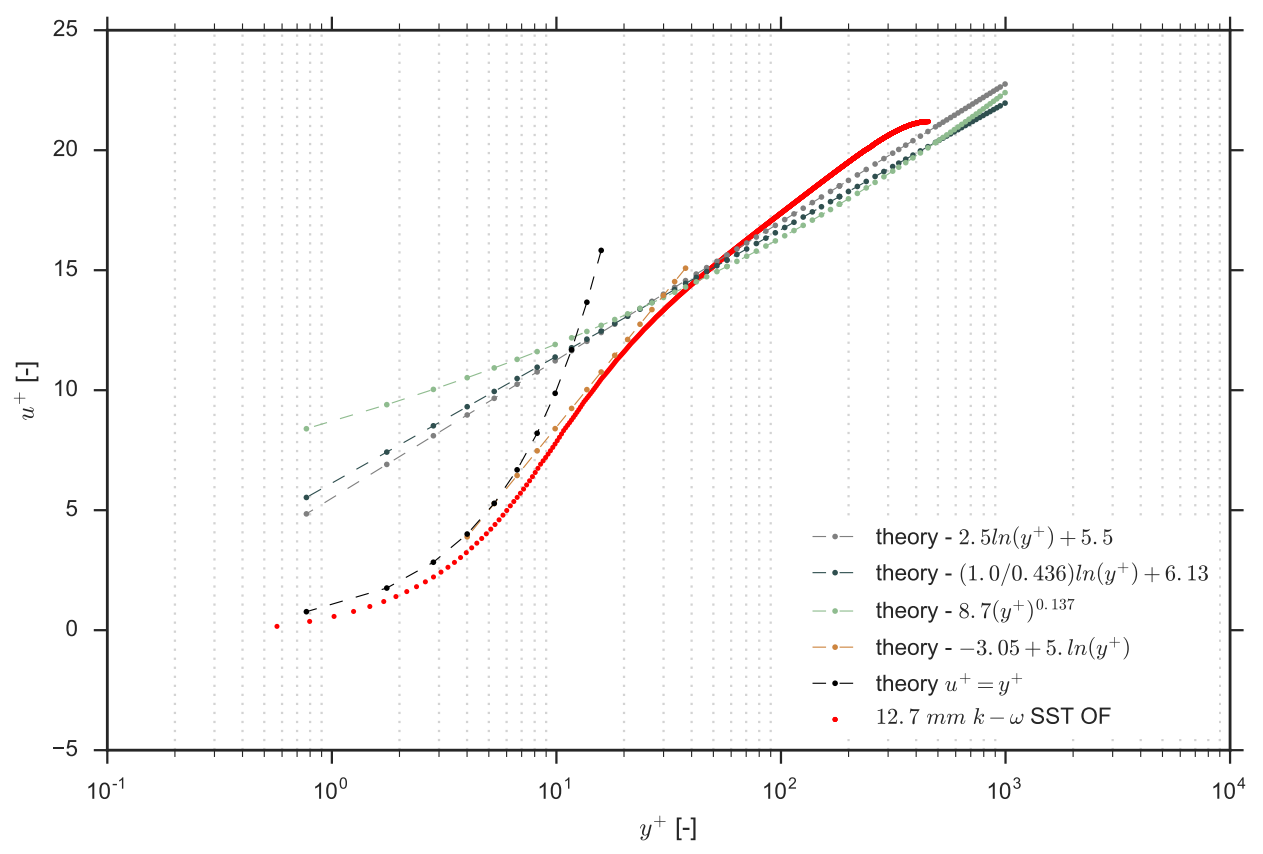

Figure 3: Non-dimensional axial velocities for various turbulence models in a $12.7 \mathrm{~mm}$ id pipe

Figure 3 compares the simulated velocity profile with the universal non-dimensional velocity profile. Several correlations have emerged from the literature to represent the regions where $y^{+}>30$ and several of the most usual ones are plotted here. It can be seen from this figure, that the computed profile is fairly close to the expected values. Note that the simulation data have been non-dimensionalized using the estimated friction velocity, which could explain the presence of a slight off-set. Figures 4 and 5 show the turbulent kinetic energy $k$ and the specific rate of dissipation $\omega$ used in all simulations. As the $k-\omega$ SST model has been selected, $\omega$ is presented instead of the commonly shown turbulent kinetic energy dispersion rate $\varepsilon=C_{\mu} k \omega$, where $C_{\mu}$ is the constant used in the $k-\varepsilon$ model (often equal to 
0.09). The turbulent kinetic energy dispersion rate can indeed often be seen as an asymptotic droplet diffusivity value for droplets that closely follow the flow (Paras \& Karabelas, 1991). Additional details are provided in Section 4.2.

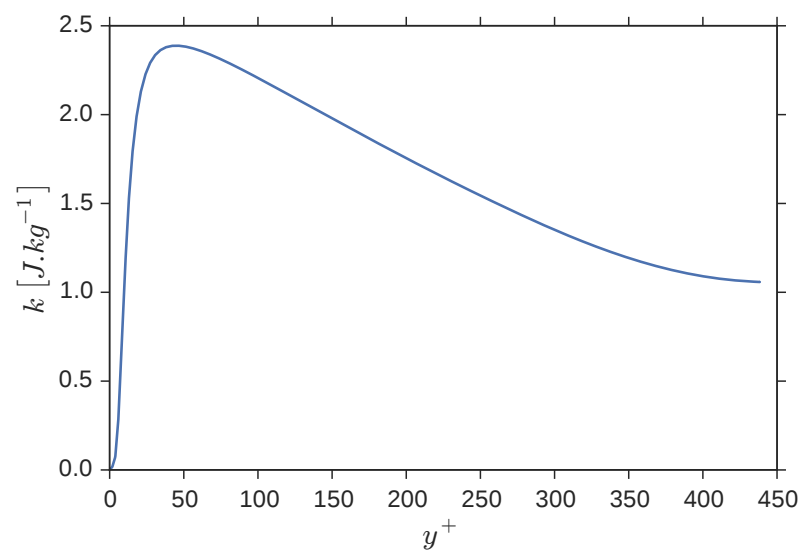

Figure 4: $k$ in $J / k g$ along $y^{+}$

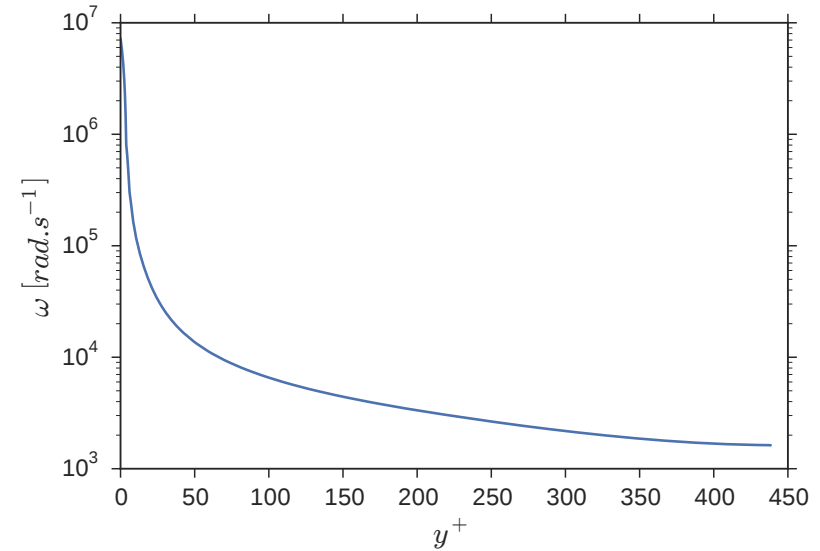

Figure 5: $\omega$ in rad/s along $y^{+}$

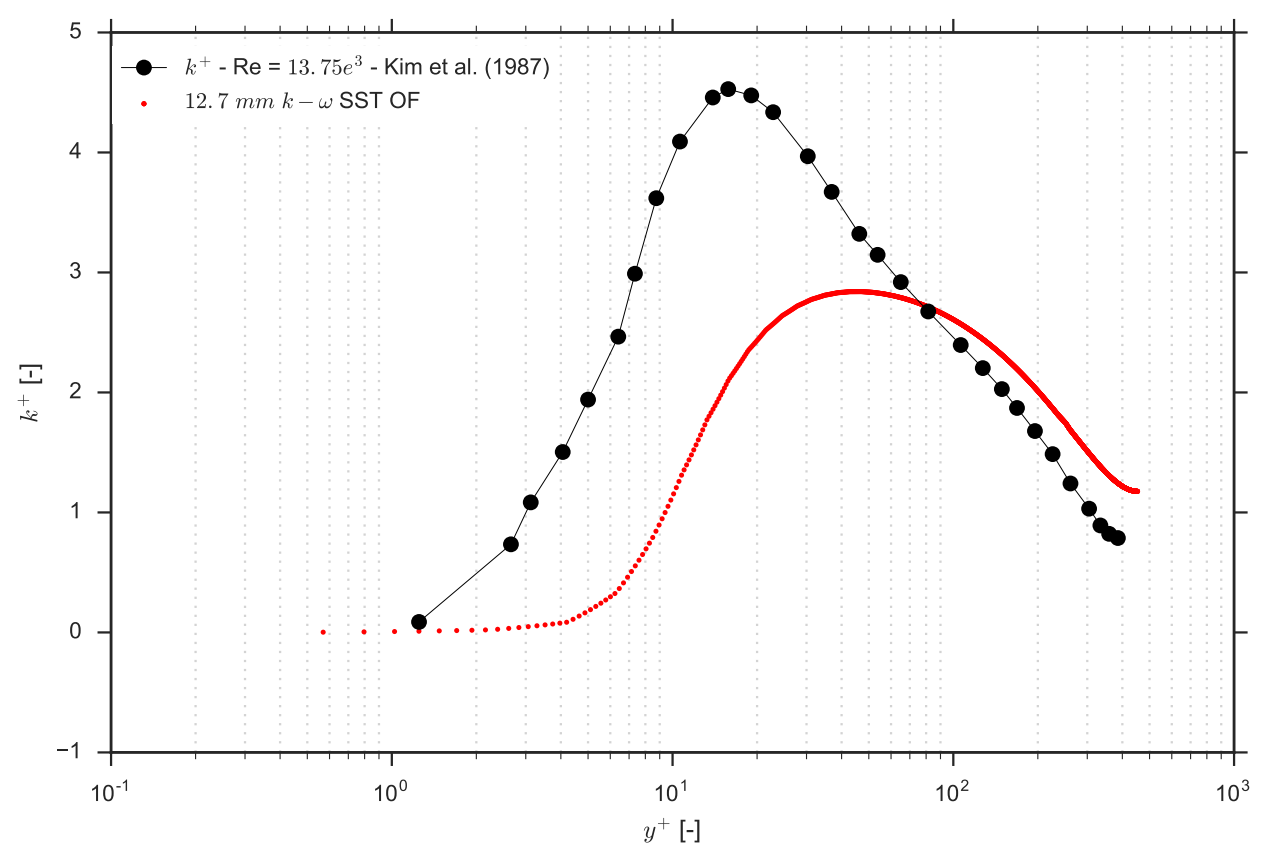

Figure 6: Dimensionless turbulent kinetic energy for various turbulence models in a $12.7 \mathrm{~mm}$ id pipe

Figure 6 compares the computed non-dimensional turbulent kinetic energy with results from Kim et al. (1987). The differences (in amplitude and peak abscissa) are expected as the $k-\omega$ SST turbulence model has been reported to show this behaviour (Hrenya et al., 1995; Kalitzin et al., 2005). Despite all these limitations, successful predictions of droplet transport have been achieved with similar methods (Matida et al., 2000).

When the flow has converged, droplets are injected in the gas core for one second to ensure all of them have either deposited or have left the domain. To match experiments from Liu \& Agarwal (1974), the disperse phase considered 
here is olive oil $\left(\rho_{p}=920 \mathrm{~kg} / \mathrm{m}^{3}\right)$ with droplets diameters ranging from $1 \mathrm{~nm}$ to $68.5 \mu \mathrm{m}$. The evolutions of three specific drop sizes, representative of the whole range of behaviours, have been specially monitored. As low droplet concentrations are present, it is assumed that droplets do not interact with the flow. A one-way coupling is therefore applied.

\section{Lagrangian conditions}

\subsection{Droplet motion equations}

The transport of particle, given by Equation 2, is called the BBO, i.e. the Boussinesq, Basset and Oseen equation:

$$
\begin{aligned}
m \frac{d u_{p_{i}}}{d t}=m g_{i} & +\underbrace{\mathcal{V}\left(-\frac{\partial p}{\partial x_{i}}+\frac{\partial \tau_{i j}}{\partial x_{j}}\right)}_{\text {undisturbed flow }}+\underbrace{3 \pi \mu_{p} d_{p}\left[\left(u_{f}-u_{p}\right)+\frac{d_{p}^{2}}{24} \frac{\partial^{2} u_{f_{i}}}{\partial x_{j} \partial x_{j}}\right]}_{\text {steady state drag }} \\
& +\underbrace{\frac{1}{2} \rho_{f} \mathcal{V} \frac{d}{d t}\left[\left(u_{f}-u_{p}\right)+\frac{d_{p}^{2}}{40} \frac{\partial^{2} u_{f_{i}}}{\partial x_{j} \partial x_{j}}\right]}_{\text {virtual mass term }}+\underbrace{\frac{3}{2} \pi \mu_{f} d_{p}^{2} \int_{0}^{t}\left[\frac{d / d \tau\left(u_{f_{i}}-u_{p_{i}}+d_{p}^{2} / 24 \times \nabla^{2} u_{f_{i}}\right)}{\pi v(t-\tau)^{1 / 2}}\right] d \tau}_{\text {Basset term }}+F_{L_{i}}+F_{i},
\end{aligned}
$$

where $\mathcal{V}$ represents the particle volume, $F_{L_{i}}$ the lift force and $F_{i}$ any other force unaccounted for. The BBO equation can be simplified based on the following assumptions:

- The droplet volume is sufficiently small so that the virtual mass term can be neglected,

- The density of the particles is much higher than the density of the carrier fluid: $\rho_{p} \gg \rho_{f}$.

A simple equation can usually be retained to describe the particle motion in the core flow:

$$
\frac{d u_{p}}{d t}=\underbrace{\frac{u_{f}-u_{p}}{\tau_{p}}}_{\text {Drag effects }}+\underbrace{f}_{\text {other forces }}
$$

where $\tau_{p}$ is the particle response time defined by:

$$
\tau_{p}=\frac{4}{3} \frac{\rho_{p} d_{e q}^{2}}{\mu_{G}} \frac{1}{C_{D} \operatorname{Re}_{p}},
$$

with $d_{e q}$ the aerodynamically equivalent particle diameter and $C_{D}$ the drag coefficient. For liquid drops, an alternative $\tau_{p}$ is used:

$$
\tau_{p}=\frac{4}{3} \frac{\rho_{p} d_{e q}^{2}}{\mu_{G}} \frac{C_{c}}{C_{D} \mathcal{R} e_{p}}\left(\frac{1+\left(\frac{\mu_{f}}{\mu_{p}}\right)}{1+\left(\frac{2 \mu_{f}}{3 \mu_{p}}\right)}\right) .
$$

The particle response time $\tau_{p}$ expresses the ability for a particle to follow the flow. Equation 3 is valid when the flow seen by the particle has a low Reynolds number. In this regime, Stokes flow approximations apply $\left(C_{D}=24 / \mathcal{R}_{p}\right)$ and the drag force is proportional to the relative velocity $u_{f}-u_{p}$. Near-wall corrections from Ahmadi \& McLaughlin (2008) have been applied with the Faxen correction defined as:

$$
C_{D} \mathcal{R} e_{p}=24\left[1-\frac{9}{16}\left(\frac{d_{p}}{2 h}\right)+\frac{1}{8}\left(\frac{d_{p}}{2 h}\right)^{3}-\frac{45}{256}\left(\frac{d_{p}}{2 h}\right)^{4}-\frac{1}{16}\left(\frac{d_{p}}{2 h}\right)^{5}\right]^{-1},
$$

where $h$ is the distance from the particle center to the nearest wall. For particles of size comparable to the carrier fluid mean free path (or smaller), the Brownian motion has to be taken into account along with the drag slip factor of Cunningham, which depends mainly of the mean free path. The following forces have been used, modified or implemented in OpenFOAM to compute the motion of the particles: 
- Drag force that accounts for sphere drag with the Cunningham factor auto-calculated with medium properties, near wall corrections and valid for droplet-based Reynolds numbers less than 1 (Stokes regime),

- Gravity effects,

- Saffman-Mei lift force for spheres (Pang \& Wei, 2011),

- Virtual mass effects,

- Pressure gradient force,

- Brownian motion, providing the mean free path $\lambda$ or the medium molecule inter-spacing.

The effects of lift can be important (Gupta \& Pagalthivarthi, 2006); the lift force should ideally include the effects of lift due to particles rotation. However, for the sake of simplicity, no droplet rotation was assumed in the current simulations.

The numerical integration commonly used to solve Equation 3 is detailed in Graham \& James (1996). The instantaneous particle velocity $u_{p}$ includes the particle fluctuation $u_{P}^{\prime}$, which arises from the turbulent dispersion influencing the droplets, see Section 3.2.

The dimensionless particle relaxation time defined as:

$$
\tau_{p}^{+}=\frac{\tau_{p}\left(U^{*}\right)^{2}}{v_{f}}
$$

is used to characterize the droplet behaviour independently of the flow conditions. Since only particles diameters vary in the current study, their behaviour can be directly associated with their size. For small droplets, where $\tau_{p}^{+}$is less than 0.3, the Brownian motion, the Stokes-Cunningham drag, and the thermophoretic forces are important. For medium size droplets $\left(0.3 \leq \tau_{p}^{+} \leq 20\right)$, Stokes drag, thermophoretic, lift, pressure gradient, centrifugal forces, conservation of angular momentum along with the turbulent dispersion should be taken into account. For large drops, where $\tau_{p}^{+}>20$, the Stokes drag, lift, pressure gradient, centrifugal, mass added forces, conservation of angular momentum and weight should be included (Matida et al., 2000).

\subsection{Turbulent dispersion - Eddy-particle interaction}

In turbulent flows, the carrier fluid velocity influences highly the particles motion. Even if Lagrangian statistics can be obtained (Govan et al., 1989), it is often easier to obtain the Eulerian description of the main fluid fluctuations rather than the Lagrangian fluctuations of the disperse phase. Intuitively, as the particles are carried by the fluid, the Lagrangian statistics should be related to the Eulerian ones. This implies that the Lagrangian auto-correlation should converge to the Eulerian auto-correlation when a particle becomes a "fluid particle". However, for large particles or when the ratio of densities becomes important, the correlation with the Eulerian field should become more distant, as particles are more likely to have uncorrelated velocities with the flow. Based on the particle velocity field (Crowe et al., 1998), the auto-correlation function $R_{P}$ can be defined as:

$$
R_{P}(x, t)=\frac{\left\langle u_{P}(x, t) u_{P}(x+\delta x, t+\delta t)\right\rangle}{\left\langle u_{P}(x, t)^{2}\right\rangle},
$$

where $u_{P}$ denotes the particle instantaneous velocity. Considering the stationary turbulent field imposed by the flow, the particle auto-correlation function can be written:

$$
R_{P}(x, t)=R_{E}(x) \times R_{L}(t),
$$

where $R_{E}$ and $R_{L}$ express the Eulerian spatial and Lagrangian temporal auto-correlations, respectively. The determination of $R_{L}$ can be obtained in different ways. For instance, Govan et al. (1989) provide a measurement of this correlation, with the following general form:

$$
R_{L}(t)=e^{-\frac{t}{\tau}},
$$

where $\tau$ can be taken as $\tau_{P}$, the particle relaxation time. Other formulations exist, such as the Berlemont et al. (1990) one, which uses Frenkiel's family of correlation function. The determination of $R_{E}$ is obtained from the two-point auto-correlation function for homogeneous isotropic turbulence. 
Taylor (1922) developed the idea of linking the Eulerian turbulence statistics to the Lagrangian turbulent dispersion for a homogeneous isotropic turbulent flow, see also Dosio et al. (2005) and Koeltzsch (1998). Taylor was thus able to determine the variance of the particle displacement in any direction and for small Stokes number particles:

$$
\operatorname{var}\left(x^{r}\right)(t)=2 \sqrt{\operatorname{var}\left(u^{\prime}\right)} \int_{0}^{t} \int_{0}^{t^{\prime}} R_{L}^{r}(\tau) d \tau d t^{\prime}= \begin{cases}\overline{u^{\prime 2}} t^{2} & \text { if } t<<T_{L} \\ 2 \overline{u^{\prime 2}} T_{L} t & \text { if } t>>T_{L} \\ \text { dependent on the correlation } & \text { otherwise }\end{cases}
$$

where var expresses the variance (using a spatial average) and $u^{\prime}$ denotes the fluctuating velocity. The superscript $r$ shows that only the radial direction is considered here. This spreading result can be recovered using the Langevin equation:

$$
\frac{d u}{d t}=-\frac{u}{T_{L}}+\sqrt{\frac{2 \cdot \operatorname{var}(u)}{T_{L}}} n(t),
$$

where $n(t)$ is a Wiener process (Szabados, 2010) and $T_{L}$ is the Lagrangian integral time scale:

$$
T_{L}=\int_{0}^{\infty} R_{L}(\tau) d \tau .
$$

Once determined, the auto-correlation function can be used to alter the particle velocity (obtained from Equation 3), by adding a fluctuating part $u_{P}^{\prime}$ :

$$
u_{P}^{n+1}=u_{P}^{n} \cdot R_{P}+u_{P}^{\prime},
$$

with $u_{P}^{\prime}$ defined as:

$$
u_{P}^{\prime}=u_{e} \sqrt{1-R_{P}^{2}} \xi
$$

$\xi$ is a random number following the standard normal distribution and $u_{e}=\sqrt{2 k / 3}$, which is representative of an eddy velocity.

Other approaches to evaluate $R_{L}$ are possible. One of the most common ones is to consider the flow as a set of eddies interacting with any particle in its vicinity. This interaction generates a constant fluctuation velocity on the particle until it leaves the eddy or until the eddy dies. When this happens, another random velocity is applied to the particle. A popular scheme for this eddy interaction model is discussed in Gosman \& Ioannides (1983), where the constant eddy-lifetime is based on a linear approximation of $R_{L}$. Using the "constant Eddy lifetime model" is similar as having the following linear correlation (Graham \& James, 1996):

$$
R_{L}(\delta T)=1-\frac{\delta T}{2 T_{L}}
$$

The time of interaction, i.e. the laps of time during which a particle keeps the same fluctuation, can be written as:

$$
T_{\text {interaction }}=\min \left(\tau_{c}, \tau_{e}\right),
$$

where $\tau_{c}$ is the crossing time and $\tau_{e}$ the eddy-lifetime:

$$
\tau_{e}=2 T_{L},
$$

with $T_{L}$ the Lagrangian integral time scale:

$$
T_{L}=C_{L} \frac{k}{\varepsilon} .
$$

Different values of time scale constant $C_{L}$ can be used, depending on the type of turbulence model selected. In most cases, a value around 0.32, as proposed by Hinze (1975), is suitable. Note however that the FLUENT (2014) manual specifies a default value $C_{L}=0.15$ and advises to apply $C_{L}=0.30$ when using the Reynolds stress turbulence model.

OpenFOAM assumes that the Lagrangian integral time scale $T_{L}$ is zero at the walls. However, it has been proven that this assumption is not valid (Bocksell \& Loth, 2006). Thus, for $y^{+}<5$, a constant adjustable value $T_{L}^{+}$is usually 
taken around 2.5. For $y^{+}>100$ the description from Equation 19 applies. For $100<y^{+}<5$, a quadratic correlation such as the one suggested by Kallio \& Reeks (1989) and applied in this work, can be used:

$$
T_{L}= \begin{cases}2.3\left(\sqrt{\frac{v_{f}}{\epsilon}}\right)_{\text {wall }} & \text { if } y^{+} \leq 5 \\ \left(7.122+0.5731 \times y^{+}-0.00129 \times y^{+2}\right)\left(\sqrt{\frac{v_{f}}{\epsilon}}\right)_{\text {wall }} & \text { if } 5<y^{+}<100 \\ C_{L} \frac{k}{\varepsilon} & \text { otherwise }\end{cases}
$$

Note that there is a discontinuity for $y^{+}=5$ in the model presented here. However, this discontinuity does not affect much the results. $T_{L}$ could have been made continuous, for instance, with a quadratic interpolation, as proposed by Lecrivain \& Hampel (2012).

As stated previously, a particle will follow the same velocity fluctuation until the eddy dies (interaction time greater than eddy lifetime) or until the drop leaves the eddy. When the particle leaves the eddy before the end of the eddy lifetime, which is expected to be more frequent for "large" particles, it will follow a new velocity fluctuation. The "crossing time" $\tau_{c}$ is the time for a particle moving at the eddy relative velocity, to travel at the characteristic length of the eddy $L_{e}$ :

$$
\tau_{c}=-\tau_{p} \ln \left(1-\frac{L_{e}}{\tau_{p}\left|u_{f}-u_{p}\right|}\right),
$$

with

$$
L_{e}=C_{\mu} \frac{k^{3 / 2}}{\varepsilon} .
$$

Using Equations 18 and 21, the local particle turbulent interaction time can be evaluated from Equation 17.

The models described above are all based on the turbulent kinetic energy. This approach can show limitations for cases where the turbulence is anisotropic (Kallio \& Reeks, 1989). Although not applied for this work, enhanced models use the Reynolds tensor components. For a numerical approach, this requires the use of a Reynolds Stress model or the use of the Boussinesq approximation. For pipe flow studies, it is a common practice to use the radial component and to define a radial Lagrangian integral time scale such as in Tian \& Ahmadi (2007):

$$
T_{L_{r}}=C_{2} \frac{\overline{v^{\prime} v^{\prime}}}{\varepsilon},
$$

where $C_{2}$ is, according to Matida et al. (2000), close to 1 .

\subsection{Diffuse dispersion - Brownian motion}

The Brownian motion is the main actor in the diffuse dispersion of droplets. Any molecule having a temperature, experiences a small random motion or oscillations. For particles small enough to see the carrier fluid as a discontinuous phase of agitated particles, the Brownian force may exist. This force results from the collisions of such fluctuating particles. A common formulation can be written, considering the vector $F_{b}$ of components $F_{b_{i}}$ :

$$
F_{b_{i}}=\zeta_{i} \sqrt{\frac{\pi S_{0}}{\Delta t}},
$$

where $\zeta_{i}$ are zero-mean, unit-variance-independent Gaussian random numbers and $S_{0}$, the spectral density, defined as:

$$
S_{0}=\frac{216 v k_{B} T}{\pi \rho_{F} d_{p}{ }^{5}\left(\frac{\rho_{P}}{\rho_{F}}\right)^{2} C_{c}},
$$


with the Cunningham slip correction factor:

$$
C_{c}=1+\frac{2 \lambda}{d_{p}}\left(1.257+0.4 e^{-1.1 d_{p} / 2 \lambda}\right)
$$

When a particle no longer sees the carrier fluid as a continuous medium, the "no-slip" condition stops being valid and the Cunningham slip factor is used to correct this.

The diffusion process has been characterized by Einstein (1905) as well as the square root of the geometric mean of the squares of the displacements in a direction, as it follows:

$$
\sqrt{\overline{x^{2}}}=\sqrt{2 D t}
$$

where $D$ is the diffusion coefficient. To test the Brownian motion model implemented in OpenFOAM, a droplet size distribution has been chosen to include sub-micron-particles, with droplets ranging from $1 \times 10^{-9} \mathrm{~m}$ to $68 \times 10^{-6} \mathrm{~m}$.

\section{Lagrangian simulation results}

\subsection{Dispersed flow results}

Ten thousands mono-dispersed droplets have been released from the center of the inlet section of the vertical pipe with the flow going downwards. This injection has been repeated for each particle size considered. This number of particles injected reduce the computational cost of the Lagrangian simulation, without impacting much the averaged deposition velocity (Lai \& Cheng, 2007). For the sake of clarity and to limit extensive post-processing, only results from three representative drop sizes are discussed in the following section. The particle-wall interaction is set to stick: once the distance between the particle center and the wall is equal to the particle radius, the particle velocity is set permanently to zero. Furthermore, there is no re-injection of parcels once a particle deposits. Due to the low number of parcels and because particles do not bounce on the walls, the number of parcels decreases with the distance to the injection point. Injecting such a low number of particles (10 000) in the domain has been motivated by three major points. First, this allows a direct comparison with the work of Matida et al. (2000), who used the same number of particles. Secondly, it appeared interesting to establish the minimum number of parcels to inject from the center of the inlet section (farthest distance to the walls) to obtain reliable results (or when the deposition velocity becomes independent of the number of parcels injected). Finally, based on the number of parcels injected and their initial location, the resulting concentration field could be analysed.

\subsubsection{Cross-section droplet statistics}

The dispersion is a measure of the droplet cloud spreading in the pipe, but could also be seen as the evolution of the droplet cloud position moments in time and space. Being able to describe the droplet dispersion renders the prediction of the droplet concentration field possible and therefore the droplet deposition. Mechanisms primarily important in the droplet isothermal dispersion are the Brownian motion, the turbulent dispersion and the convective transport. There are several ways to describe dispersion, either spatial, temporal or based on a spectral analysis. The spatial and temporal evolutions of the dispersion are discussed here.

The positions of the droplets of size $0.5 \mu \mathrm{m}$ and $7.8 \mu \mathrm{m}$ and their associated radial distribution have been evaluated at various axial sections of the pipe. The objective was to establish a general way of describing the shape of the transient and steady state dispersion patterns for a large range of droplet sizes. It is important to find a universal radial distribution shape, which would be valid to describe the whole evolution of the dispersion. The evolution of the first three statistical moments are reported in the next section. Note that if the general expression of the radial distribution is known and is combined with the reported moment variations, the droplet concentration field (and resulting deposition) can be determined at every point in space and time.

The probability for a droplet to be in a radial interval is a random variable. It can be deduced by counting drops in bins, distributed along the pipe radius. 


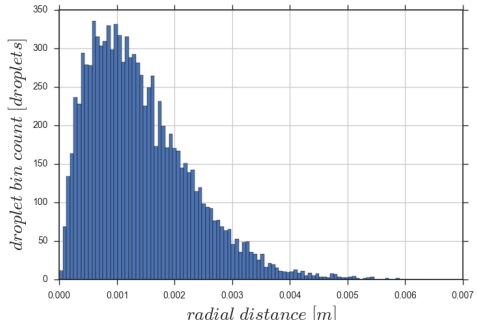

(a) early dispersion $x=0.14 m$

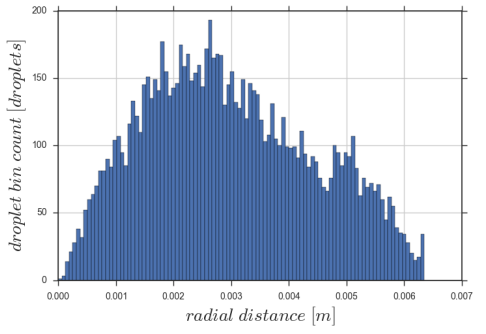

(b) medium dispersion $x=1.008 \mathrm{~m}$

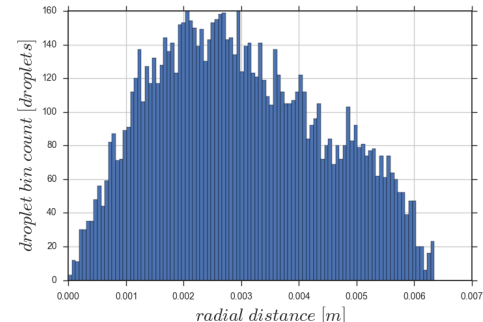

(c) late dispersion $x=2.54 \mathrm{~m}$

Figure 7: Spatial evolution of the radial dispersion - $0.5 \mu \mathrm{m}$ droplets

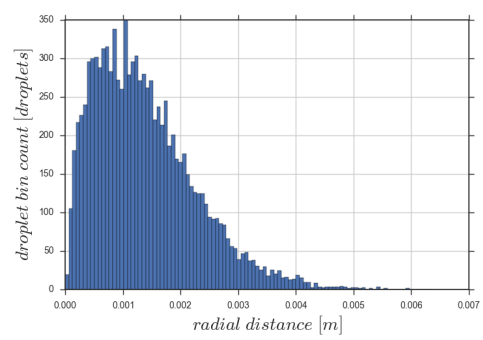

(a) early dispersion $x=0.14 m$

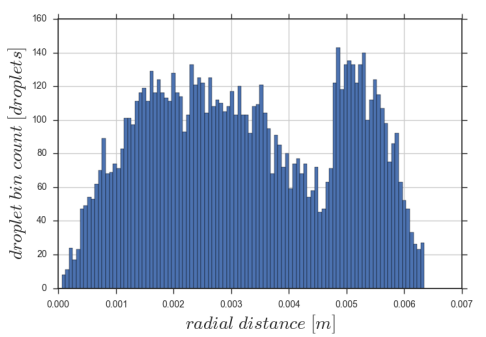

(b) medium dispersion $x=1.008 \mathrm{~m}$

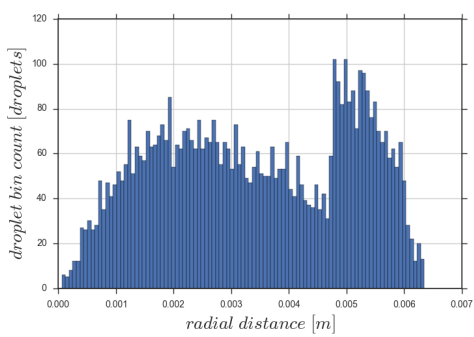

(c) late dispersion $x=2.54 \mathrm{~m}$

Figure 8: Spatial evolution of the radial dispersion - $7.8 \mu \mathrm{m}$ droplets

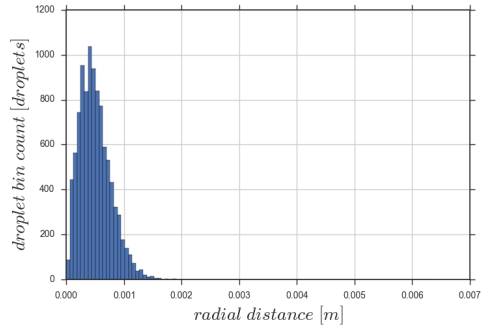

(a) early dispersion $x=0.14 m$

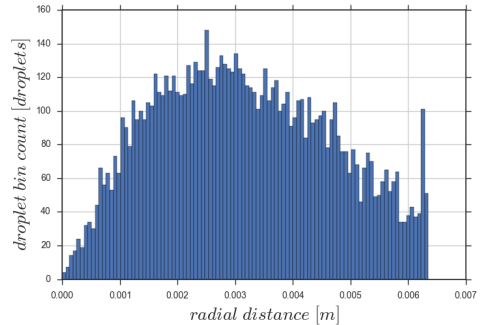

(b) medium dispersion $x=1.008 \mathrm{~m}$

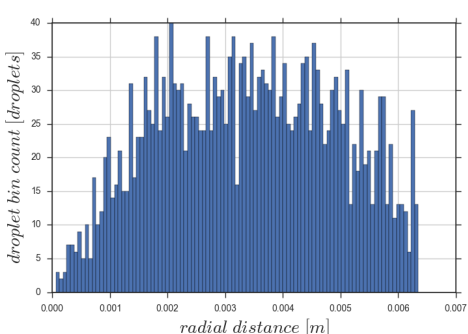

(c) late dispersion $x=2.54 \mathrm{~m}$

Figure 9: Spatial evolution of the radial dispersion - $68.5 \mu \mathrm{m}$ droplets

Histograms displayed in Figures 7 to 9 were produced by generating a $0.0255 \mathrm{~m}$ wide clip of the droplet pathlines and filtering them to keep one point only per droplet. All early dispersion profiles in these figures are similar, even though they do not necessarily develop at the same speed. After some time in the dispersion process, the radial distribution begins to be dependent on the droplet size.

Three zones are visible when the equilibrium state is reached: i.) a common dispersion shape, up to $0.005 \mathrm{~m}$, ii.) a drop size dependent shape between $0.005 \mathrm{~m}$ and $0.006 \mathrm{~m}$ and iii.) a wall droplet build-up, common to all droplet sizes, but with various amplitudes. These regions seem consistent with the 3 layers described by Equation 20.

No satisfactory universal distribution has been found yet to represent at once all those states. A more detailed study should be devoted to that purpose only. A linear combination of several distributions will allow a finer and complete description of the previously described evolutions. However, such approach has not been investigated here. 
The polar representation of the droplets positions illustrates how a radial distribution is represented in a disk area. Since 10,000 drops only are injected in the domain and since they stick to the wall when they reach it, too few of the $68.5 \mu \mathrm{m}$ drops are present near the outlet section of the pipe (most of them deposit between 1 and $2 \mathrm{~m}$ ), rendering the analysis very difficult for droplets of this size. However, the analysis has been performed for the medium and small drop sizes considered here. Figure 10 shows the resulting polar representation from a profile similar to Figure 7(c) for the $0.5 \mu \mathrm{m}$ droplets. The associated droplet surface concentration is represented by an histogram on Figure 11 . Similar plots are obtained for the $7.8 \mu \mathrm{m}$ droplets, see Figures 12 and 13.
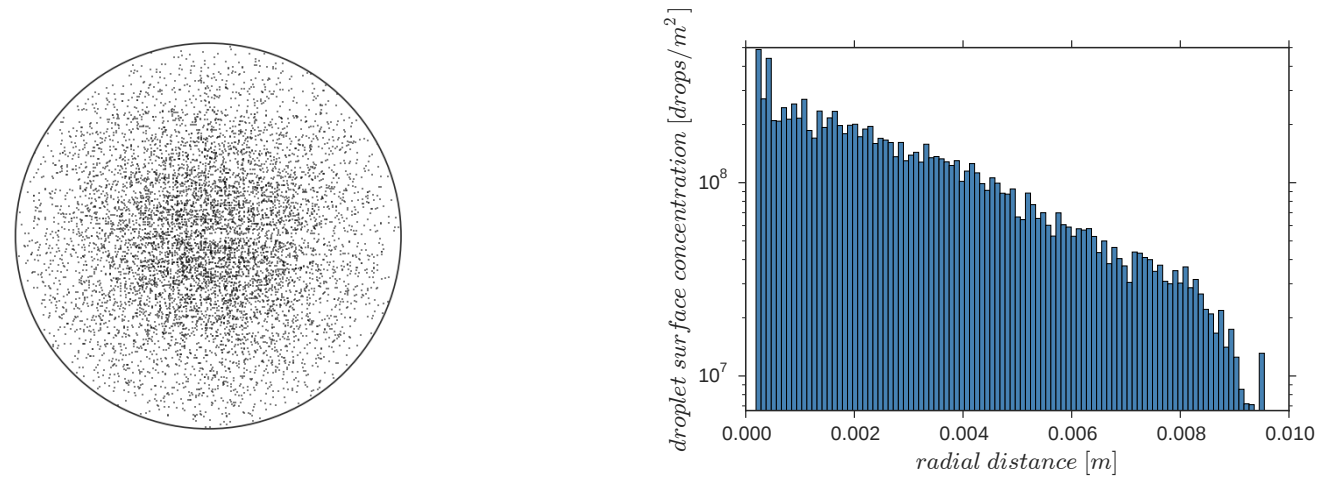

Figure 10: 0.5 microns droplets positions at the pipe outlet section

Figure 11: Radial surface concentration: 0.5 microns
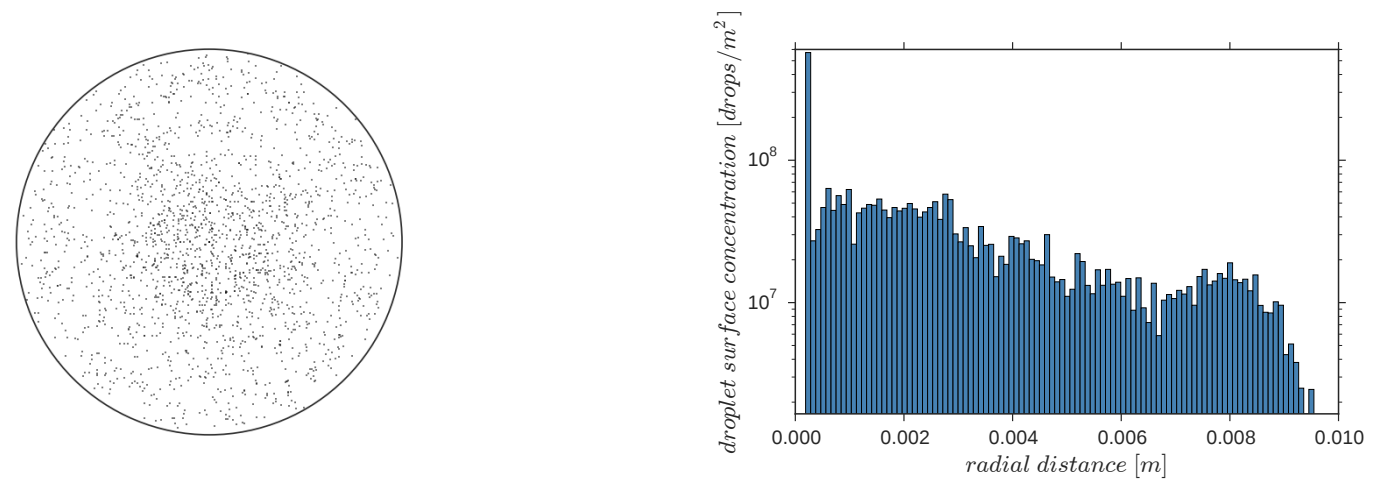

Figure 12: 7.8 microns droplets positions at the pipe outlet section

Figure 13: Radial surface concentration: 7.8 microns

The histograms on Figures 11 and 13 represent 100 pipe angular sectors of identical width (annuli areas). Sectors close to the center of the pipe therefore have a smaller cross-sectional area than sectors next to the walls. Each vertical bar represents the number of droplets collected in each annuli sector divided by the annuli surface. The polar representation is achieved through clipping the droplets pathlines data one centimetre far from the outlet section and projecting the mean position of each droplet pathline onto a $2 \mathrm{D}$ surface.

In all cases, droplets are mainly concentrated around the center axis of the pipe, the concentration decreasing as the annuli area increases from the pipe center to the wall.

\subsubsection{Droplet dispersion}

The previous section has described the general aspect of the cloud dispersion, particularly in its equilibrium state. Measuring the spatial and temporal evolutions of the radial dispersion moments leads to an estimate of the cloud dispersion. 
The time evolution of the moments (mean radial position of the drops for a given time), the variance (axial) and the skewness are provided in Figures 14, 15 and 18. The continuous lines displayed on these figures represent the exponential fit curves for the data obtained numerically. A similar general behaviour can be seen in all plots: a starting transient phase followed by a relatively flat profile. The spatial evolutions shown in Figures 16, 17 and 19 display a straight pattern, less noisy than the time profiles. It can be seen that the droplet dispersion needs some time and some distance to reach an equilibrium state characterized here by the moments being constant. For the drop sizes investigated, a time of $0.2 \mathrm{~s}$ and a distance of about $3 \mathrm{~m}$ are necessary to get a fully established droplet dispersion. As expected, the dispersion of the largest droplets require more time to reach an equilibrium state. Near the end of each time profile, the data oscillate highly. Such variation is not clearly visible in the spatial distributions. This final de-coherence can be explained by the lack of particles near the end of the simulation, making any statistical analysis unreliable at the end of each simulation.

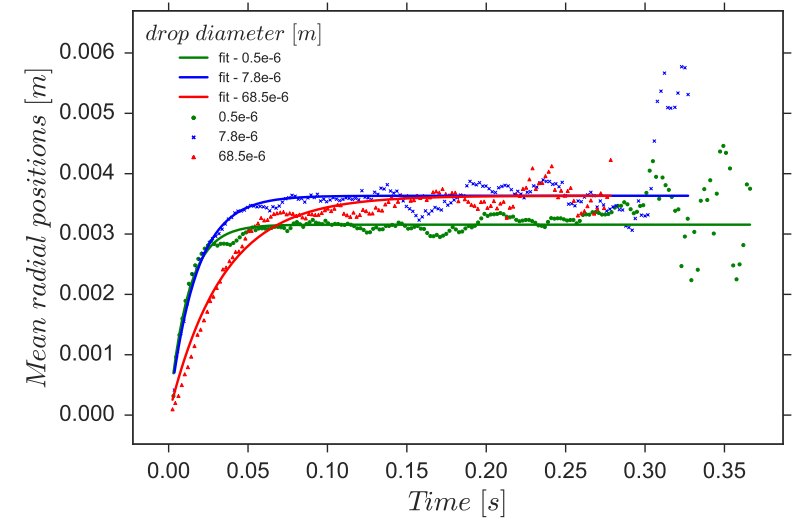

Figure 14: Time evolution of the mean radial position of droplets

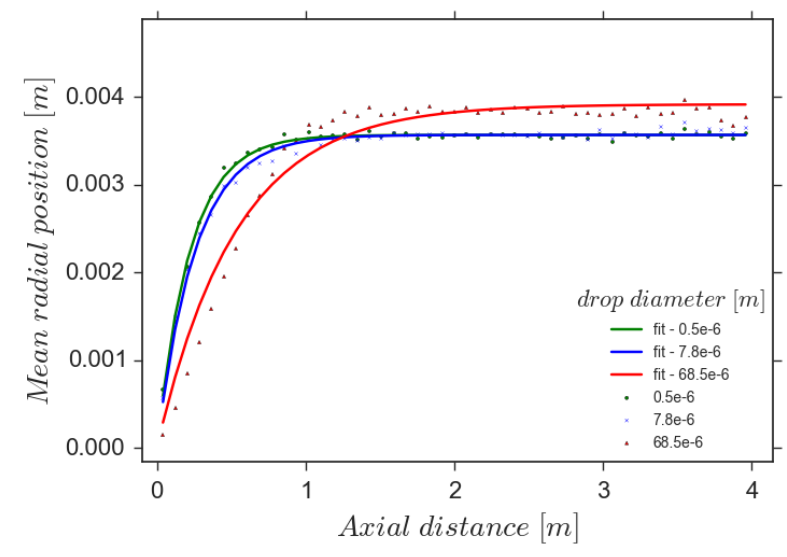

Figure 16: Spacial evolution of the mean of the radial positions

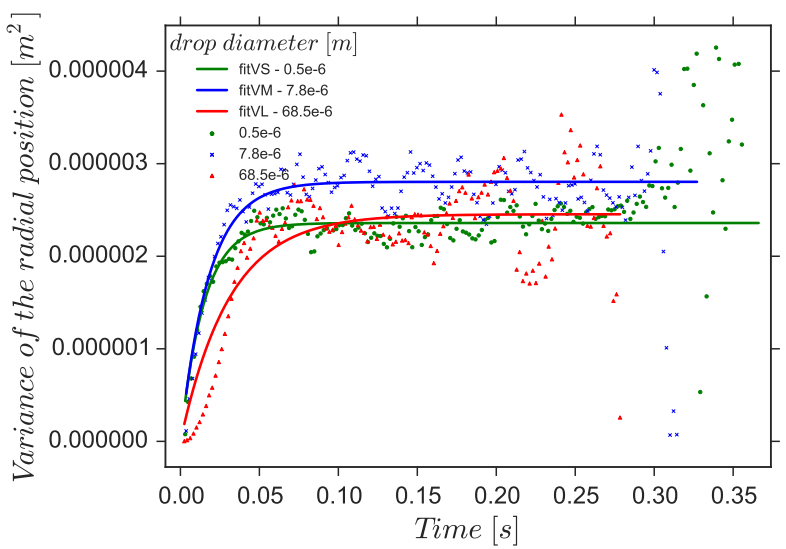

Figure 15: Time evolution of the variance of the radial position of droplets

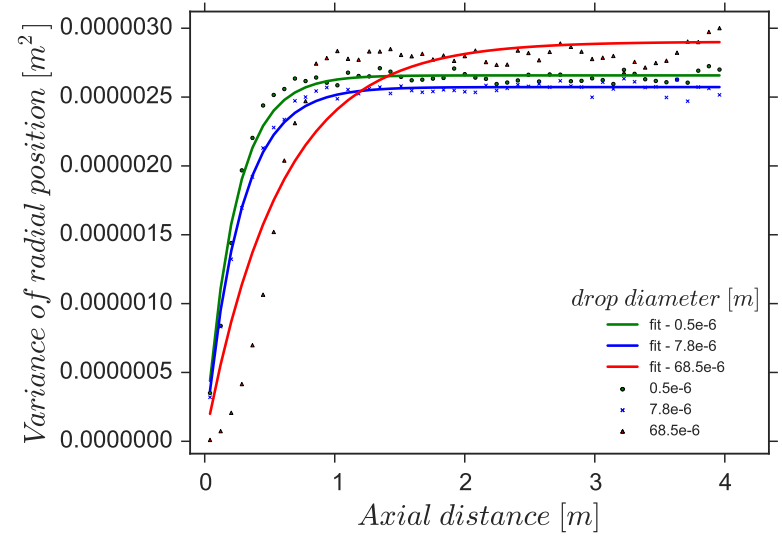

Figure 17: Spacial evolution of the variance of the radial positions 


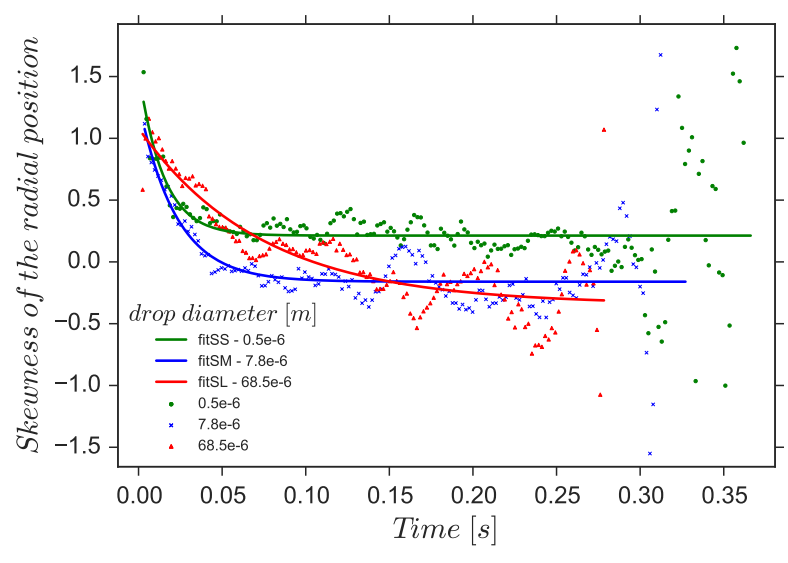

Figure 18: Time evolution of the skewness of the radial positions

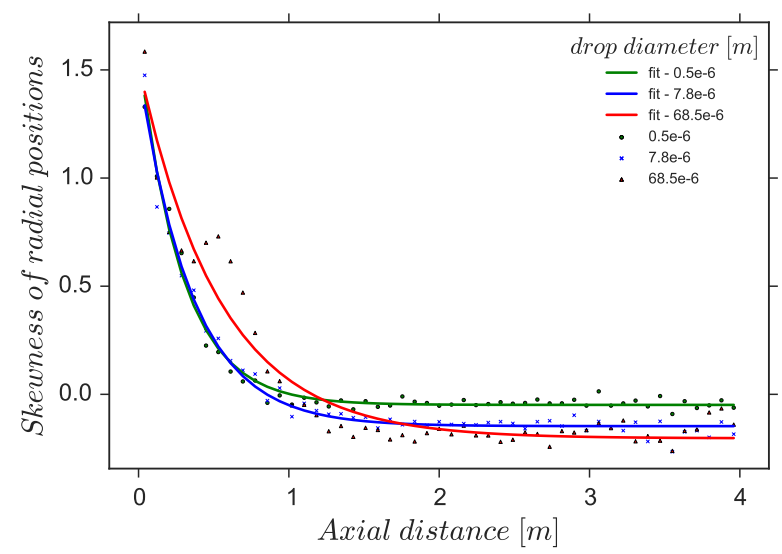

Figure 19: Spacial evolution of the skewness of the radial positions

To match the data plotted from Figure 14 to 19 and create a statistical model, exponential-based functions can be used; they are represented by plain lines on the figures. It is supposed that the variance and the mean variation follow an exponentially growing function $A\left(1-\exp \left(-t / \tau_{A}\right)\right)$ while the skewness is assumed to follow a decreasing exponential $B \exp \left(-t / \tau_{B}\right)+C$. Such exponential functions can be established, based on the overall shape of the evolution of the moments (variance, mean variation and skewness) which follow an exponential pattern. Furthermore, intuitively, an exponential law can generally be used in phenomena where an equilibrium value is asymptotically reached. The corresponding fitted values of $A, B, \tau_{A}, \tau_{B}$ and $C$ are written in Table 2.

Table 2: Fitting function parameters $A, B, \tau_{A}, \tau_{B}$ and $C$

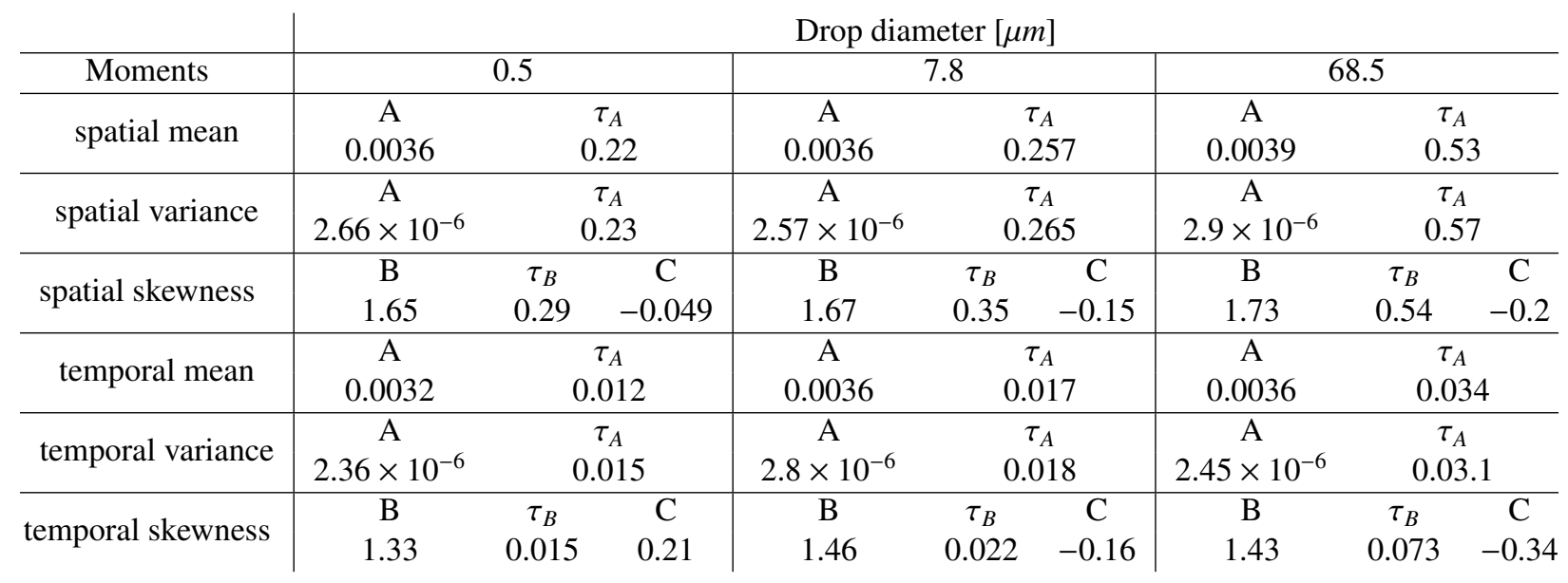

Figure 20 shows the different existing states of dispersion and deposition for the injection. In Sector 0, the droplet cloud spreads quickly but does not reach the walls. Sector 1 depicts the region where the spreading has reached the walls, but is still not fully established. Droplets start depositing, but the rate of deposition has not yet reached its equilibrium state. Sector 2 represents the region where the dispersion is established and where a stable deposition velocity can be evaluated. Sector 3 may exist, depending on the flow settings. This region is present for the current simulations as all droplets are injected simultaneously and the flow conditions and droplet sizes allow such deposition. In this region, the droplet concentration decreases since most droplets have already deposited. Finally, no deposition is present in Sector 4, which corresponds to a dry wall region. This can occur as either all droplets have already 
deposited in previous regions (no drops present either in the core flow), or the dispersion pattern is such that the nearwall concentration is low and/or the overall concentration is too low to be accounted for (only a few droplets deposit).

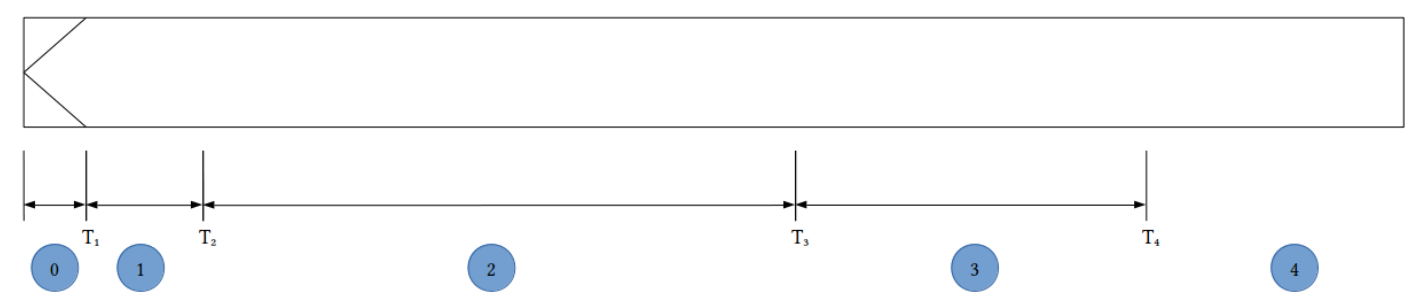

Figure 20: Evolution of the dispersion/deposition scheme

Picano et al. (2009) provided a relationship to evaluate the distance where the fully developed concentration profile in a pipe is reached:

$$
\frac{L_{\infty}}{R} \simeq 75 \tau_{p}^{+0.21} .
$$

When this formula is applied to the current cases, the fully developed droplet concentration is estimated to be reached at $0.2616 \mathrm{~m}, 0.7723 \mathrm{~m}$ and $1.92 \mathrm{~m}$ for the droplets of sizes $0.5 \mu \mathrm{m}, 7.8 \mu \mathrm{m}$ and $68.5 \mu \mathrm{m}$, respectively. These values do not seem to agree with curves shown previously on Figures 16, 17 and 19. A similar equilibrium-state concentration formula has however been determined, assuming that an equilibrium state pattern in the dispersion has been reached at the $3 \tau_{B}$ position which corresponds to $95 \%$ of the asymptotic value reached:

$$
\frac{L_{\infty}}{R} \simeq 157.388 \tau_{p}^{+0.06453} .
$$

This correlation is different from Picano's one represented graphically on Figure 21. However, Equation 28 has been established using a continuous drop injection, which differs from the drop injection in this work, and for a Reynolds number of 3,000, which is also different from the one used in this work $(R e \simeq 15,000)$. Equation 29 provides fully developed concentrations obtained after $0.83 \mathrm{~m}, 1.16 \mathrm{~m}$, and $1.54 \mathrm{~m}$, for droplets of sizes $0.5 \mu \mathrm{m}, 7.8 \mu \mathrm{m}$ and $68.5 \mu \mathrm{m}$, respectively.

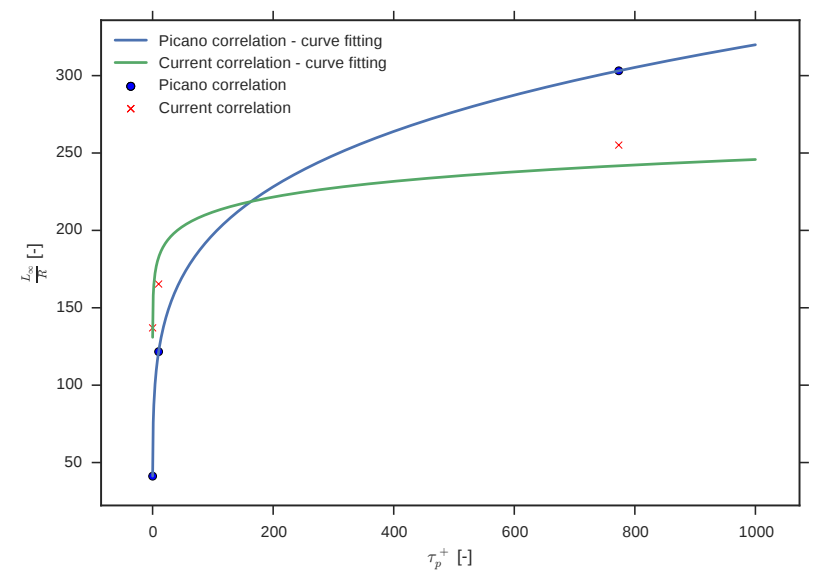

Figure 21: Correlation comparison 


\subsubsection{Deposition results and analysis}

To analyse the droplets deposition, their position and the time required for them to deposit, the impact velocities have been investigated.

The cumulative density function (CDF) and probability density function (PDF) of deposition time (which can be compared to the first-passage time density function) allow to determine how rare the initial and "dry-out" deposition in the pipe can be (along with the deposition in the other sectors). Figure 22 shows the cumulated density function obtained for each drop size separately, in the $5 \mathrm{~m}$ long pipe. This figure shows the four different sectors described previously through Figure 20. No deposition is present at the start of the simulation (Sector 0). After a laps of time, the deposition grows "exponentially" (Sector 1) to reach a region where the deposition rate is almost constant, i.e. where the slope of the curves is almost constant (Sector 2). The decrease of the number of drops decreases the deposition rate (curved region, Sector 3). Past this region, a plateau is present (more or less visible depending on the droplet size), which is due to the particles which have spent some time in the turbulent eddies or have been trapped in the boundary layer. The longer the plateau, the more sensitive a particle size to turbulence. Finally, the last region shows no deposition (Sector 4). The colored lines present above Figure 22 represents the time limits of all sectors represented in Figure 20, where each color corresponds to a particle size (color displayed in the legend).

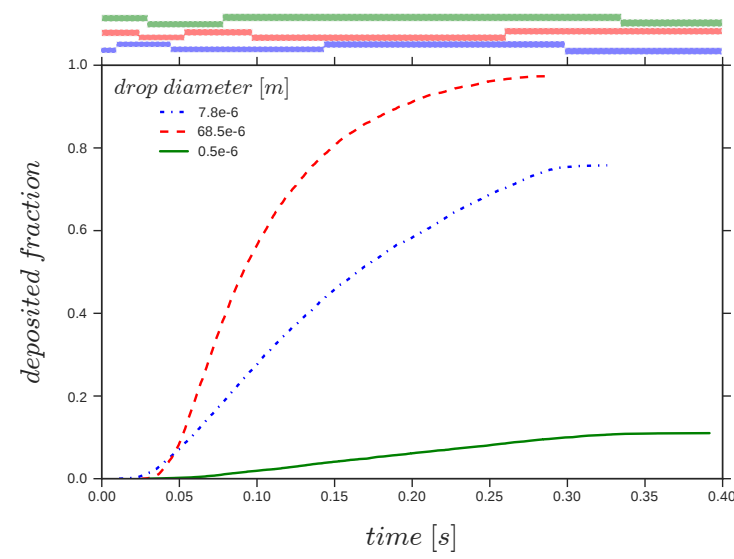

Figure 22: $C D F$ of time of deposition - deposited fraction along the time $(0.5,7.8$ and 68.5 microns $)$

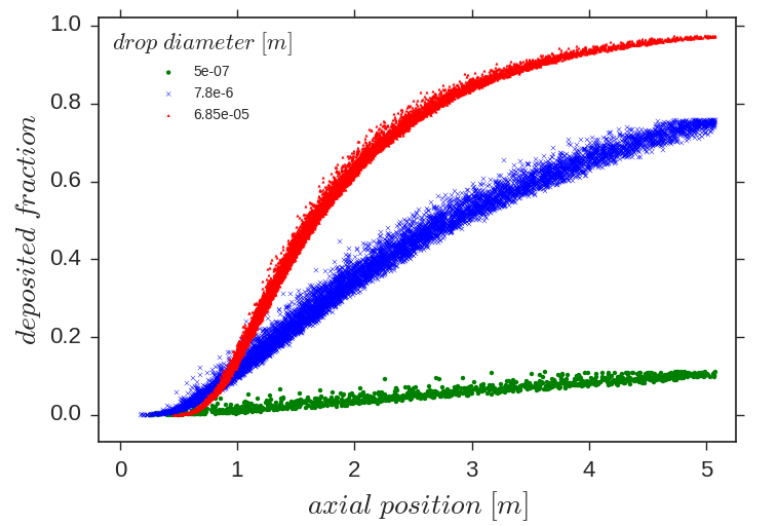

Figure 23: CDF of axial position of deposition (current simulation)

Figure 23 shows the ratio of deposited to the number of injected droplets against the axial position down the pipe. The curves follow a similar pattern as the time evolution of the same drop sizes in Figure 22. This result was expected since most drops are merely transported away by the flow. It can also be seen that for a given deposition fraction level, the deposition occurs over a certain spatial length.

The larger the drops, the faster the deposition. As shown on Figure 22, the time required for $90 \%$ of the drops to deposit is shorter for the large drops than for the small ones. Figure 23 shows that most large drops deposit between 1 and 2 meters. 


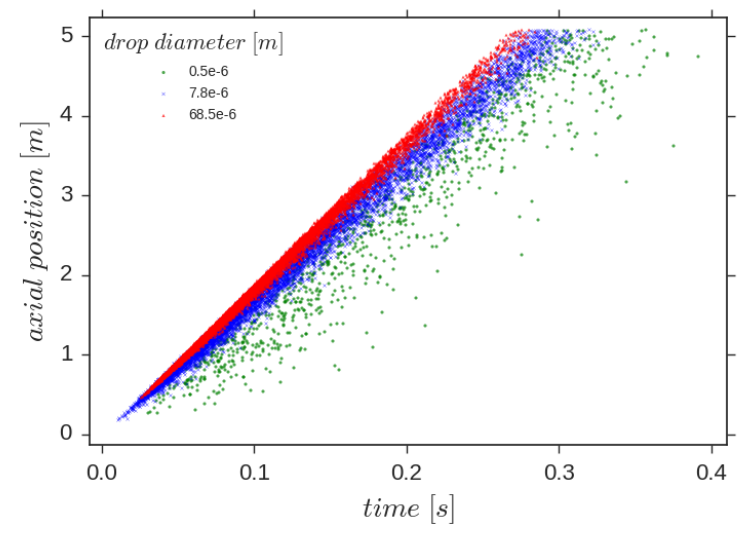

Figure 24: Correlation between the impact position and the impact time

A similar study has been performed for the spatial evolution. A strong correlation can be established between the time and the spatial evolutions of the variables, as shown in Figure 24. This correlation decreases with time and distance. The correlation, clearly visible for large drops which have a ballistic trajectory, is however less pronounced for small diffusive particles which are more likely to be affected by turbulence and Brownian motion effects. For each impact position, there is a time interval represented by a lower and an upper bound during which all the deposition occurs. This interval varies with the droplet size and the time. Far away from the inlet of the pipe, the smallest droplets will take more time to deposit while the largest droplets will deposit quickly next to the inlet section of the pipe. This remark is also valid when looking at the space interval for each droplet size: the maximum distance between two deposited droplets is larger for small droplets than for large ones.

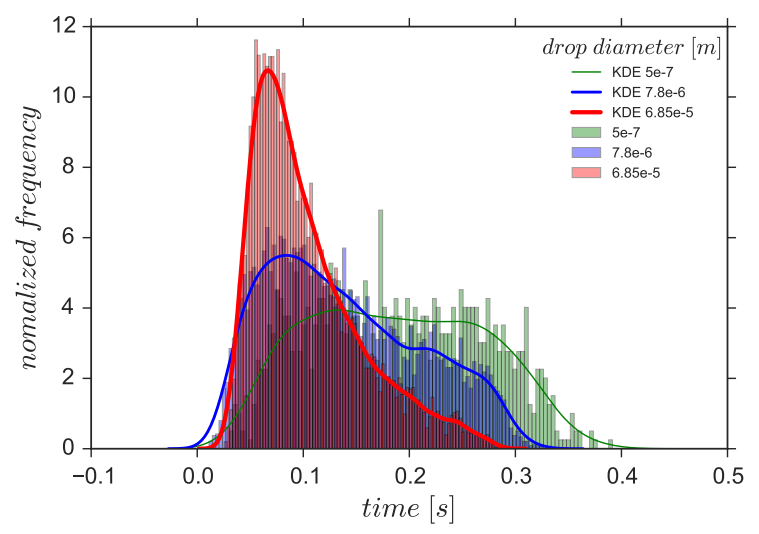

Figure 25: PDF of deposition time and their respective Kernel Density Estimators

Previous comments are further demonstrated through Figure 25 which shows the PDFs of deposition time and their respective Kernel Density Estimators. The larger the particle, the narrower the "time-bandwidth" (90\% of the deposition occurs within a small period of time). The PDFs of the large and medium size drops seems to follow an inverse Gaussian shape. For the smallest drops, the PDF seems to be more evenly spread and over a larger time range.

Most of the large $(68 \mu \mathrm{m})$ drops deposit around $0.1 \mathrm{~s}$, i.e. the statistical mode is about $0.1 \mathrm{~s}$. The mode of the $7.8 \mu \mathrm{m}$ drops is less pronounced, but also seems to be around $0.1 \mathrm{~s}$. There is however no significant mode visible for the smallest drops, which seem to deposit with the same probability between $0.1 \mathrm{~s}$ and $0.25 \mathrm{~s}$. It is well known (Kou 
\& Wang, 2003; Atiya \& M., 2005), that the "first passage time PDF" (i.e. the estimate of the probability density of time needed for a random process to cross a given level) for a jump-diffusion process with exponential jumps, is a generalized inverse Gaussian distribution. The Brownian motion with occasional turbulent jumps belongs to this family of processes; this is consistent with the PDF shown in Figure 25.

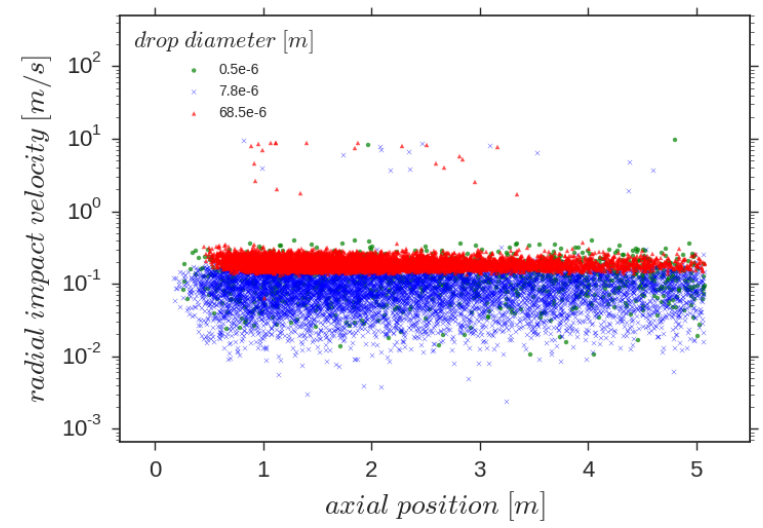

Figure 26: Radial component of the impact droplet velocity versus the axial position

Figure 26 shows the axial variation of the radial component of the particles velocity during their impact on the wall surface. The large drops have a higher value as they act like inertial particles while the $7.8 \mu m$ drops have a lower radial velocity. However, for the sub-micron particles, the radial velocity component can be as high as for the $68.5 \mu \mathrm{m}$ droplets and as small as for the $7.8 \mu \mathrm{m}$ ones. This means that the Brownian motion plays a major role here. It can also noticed that several values are outside the "main" lines: this is probably caused by drops influenced by turbulence (from higher fluid layers) which have kept their burst velocity until they reach the walls.

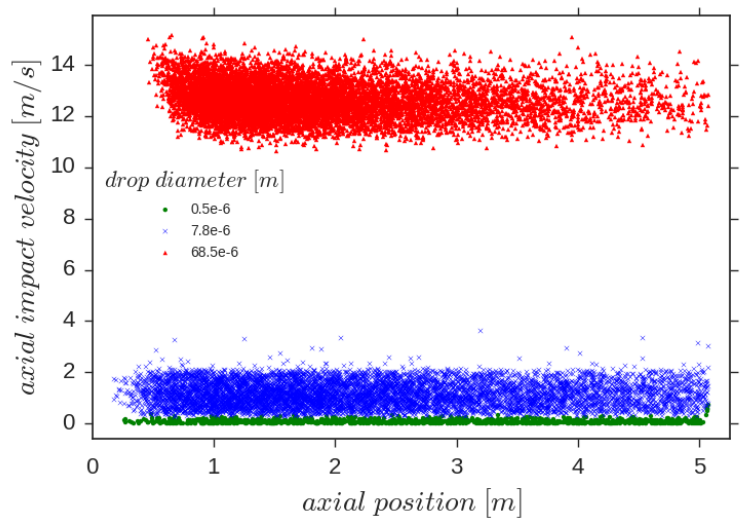

Figure 27: Axial component of the impact droplet velocity versus the axial position

Each droplet size has a distinct and restricted possible range of impact axial velocity components, see Figure 27. This velocity does not significantly change with the axial position. Note that the number of impacts decreases as drops reach the outlet section of the pipe. As stated previously, fewer droplets deposit in this region. The larger the droplets, the higher their axial impact velocity. This could again be explained by the fact that large drops acquire speed from the highest fluid layers and keep their velocity longer. On the contrary, smaller droplets adjust more easily their speed 
to the surrounding fluid velocity. The magnitude of the axial impact velocity is quite low for the sub-micron droplets and of the same order of magnitude as the radial impact velocity.

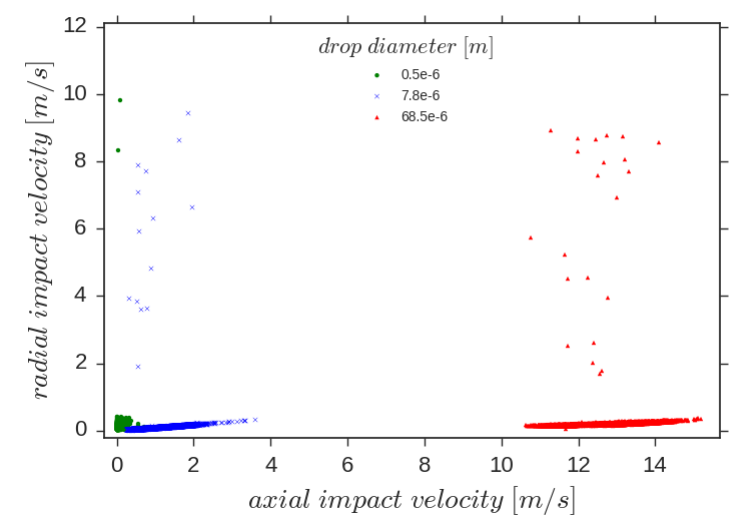

Figure 28: Radial impact velocity versus axial impact velocity

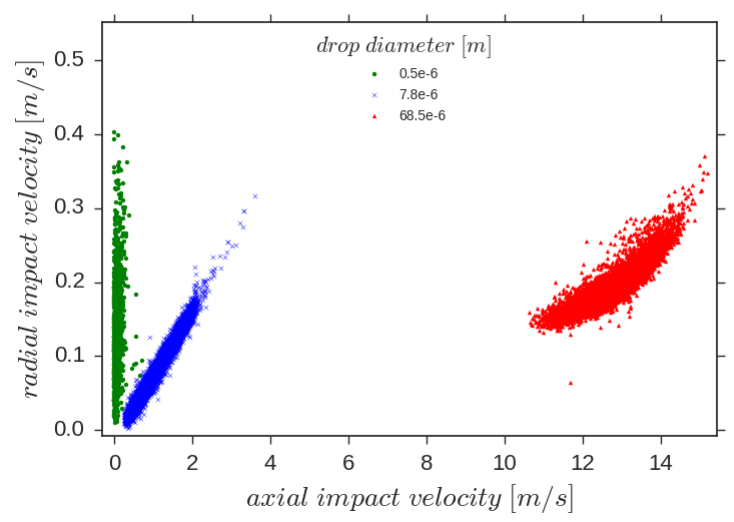

Figure 29: Detail view of Figure 28

Figures 28 and 29 provide additional information on the impact velocity. Both axial and radial velocity components are small and of the same order for the sub-micron droplets. However, it appears that such droplets are more likely to reach the wall with a velocity almost perpendicular to the wall (visible in Figure 29, where most of the points are located on the upper part of the line radial velocity = axial velocity). This would suggest that for sub-micron droplets, the main contribution to deposition is not only due to the cloud advance, but also to forces acting in the radial direction (Brownian motion and turbophoresis).

For the $7.8 \mu \mathrm{m}$ droplets, the magnitude of the impact velocity is higher than for sub micron droplets. The impact velocities are almost all aligned along a line of equation $y=0.0878 x-0.015$, where $x$ is the axial impact velocity (up to 4) and $y$ represents the radial impact velocity. This equation is valid for all droplets except for those depositing because of a turbulent event. In this case, the radial component becomes higher than the axial component (off line points on Figure 28). Droplets affected by turbulence should impact the walls with a higher velocity. Such droplets should remain unaffected by the boundary layer, they are therefore not aligned. Slower drops which have spent some time in the near-wall boundary layer are more likely to be aligned (Picano et al., 2009).

For the $68.5 \mu \mathrm{m}$ droplets, the impact velocities seem to follow an exponential profile. Several droplets are affected by turbulence, and consequently do not follow the exponential curve. However, in contrast to the $7.8 \mu \mathrm{m}$ droplets, turbulent impacts cannot make the radial component higher than the axial component.

Overall, it appears here that the near wall concentration is more highly dependent on the cloud axial advance rather than its radial dispersion, thus resulting in impact angles on the wall lower than $45^{\circ}$. In addition, the time scale of the radial dispersion is much slower (up to 100 times) than the axial convection. This means that once a set of particles has reached a given axial position, some time is required for particles at that axial position to reach the walls and reach their equilibrium state.

\subsubsection{Droplet deposition validation}

Figure 30 summarizes the equilibrium state deposition results obtained with OpenFOAM. They are compared to numerical results from Li \& Ahmadi (1993), He \& Ahmadi (1999), Matida et al. (2000), Marchioli \& Giusti (2003), to experimental data from Liu \& Agarwal (1974) and to those reported by Papavergos \& Hedley (1984). Additional numerical simulations have been performed with FLUENT (2014) for further comparison. The non-dimensional particle relaxation time $\tau_{p}^{+}=\tau_{p}\left(u^{* 2} / v\right)$ where $u^{*}$ is the friction velocity and $v$ the kinematic viscosity, is plotted in function of the non-dimensional deposition velocity $k_{p} / u^{*}$. The formula from Matida et al. (2000) has been used to 
calculate the deposition velocity:

$$
k_{p}=\frac{\overline{u_{f}} d_{t}}{4\left(x_{2}-x_{1}\right)} \ln \left(\frac{F_{1}}{F_{2}}\right)
$$

where $F_{1}$ and $F_{2}$ are the deposited fraction at two positions, $d_{t}$ is the pipe diameter, $x_{2}-x_{1}$ is the interval between the two axial positions.

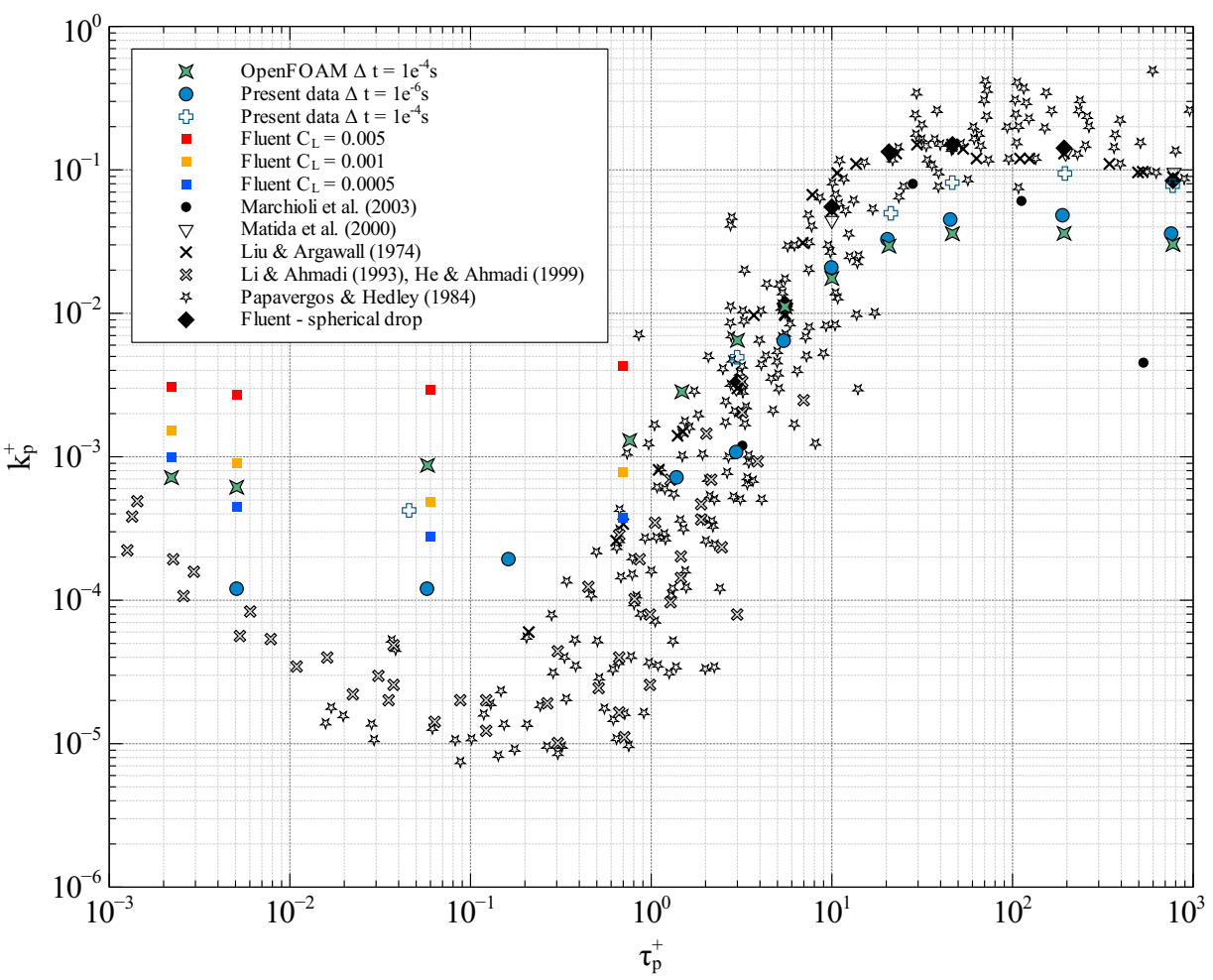

Figure 30: Deposition velocity vs. relaxation time - Simulations and literature

Two zones can be differentiated in Figure 30, when the non-dimensional particle relaxation time $\tau_{p}^{+}$is higher or lower than unity.

For the first region, when $\tau_{p}^{+} \geq 1$, results are slightly under-predicted with OpenFOAM when compared to experiments although they are consistent with a DNS study from Marchioli \& Giusti (2003). Those obtained with the commercial code FLUENT and those from Matida et al. (2000) are closer to the experimental data. However, in the contrary of OpenFOAM where the time scale constant remains constant for all droplet sizes $\left(C_{L}=0.32\right)$, this time scale constant has been determined specifically for each droplet size in FLUENT. According to the FLUENT manual, " $C_{L}$ is to be determined and is not well known". However, guidelines are provided: $C_{L}=0.15$ when applying the k-epsilon turbulence model and $C_{L}=0.30$ for the Reynolds-stress turbulence model. Tian \& Ahmadi (2007) specified that no universal value of $C_{L}$ has been found yet, but typically, a value in the range 0.2 to 0.96 has been reported in the literature for producing satisfactory results for comparison with experimental data.

For the second region, when $\tau_{p}^{+}<1$, the influence of the Brownian motion is important. Results generated with OpenFOAM are closer to experiments than those obtained with FLUENT with low $C_{L}$ values. FLUENT assumes that the turbulent integral time scale $T_{L}$ is defined everywhere as $T_{L}=C_{L} \frac{k}{\varepsilon}$, and in particular next to the walls. Applying a small $C_{L}$ value means that the near wall eddy-lifetime will be shortened, which complies with Equation 20.

Simulated results are higher than experimental and numerical evaluations reported in the literature, see Tian \& Ahmadi (2007); Papavergos \& Hedley (1984); Li \& Ahmadi (1993); He \& Ahmadi (1999) for instance. However, experimental 
measurements reported by Papavergos \& Hedley (1984) and numerical solutions from the other authors were obtained in a vertical duct, while simulated results from OpenFOAM and FLUENT are obtained in a vertical pipe.

\subsection{Particle concentration and deposition velocity}

Although previous results of dispersion and deposition have been described separately, they are related. The particle concentration should be seen as an Eulerian field which is affected by the dispersion. Knowing the concentration field, its evolution and the turbulent field, the deposition can be predicted. This can be achieved with most Eulerian formulations by means of a diffusion-convection equation. An Eulerian drift-flux model such as the one reported by Lai \& Cheng (2007) can be used to solve the evolution of concentration:

$$
\frac{\partial C}{\partial t}+\operatorname{div}\left[\left(u+V_{s}\right) C\right]=\operatorname{div}\left[\left(D+\varepsilon_{p}\right) \operatorname{grad}(C)\right]+\text { Sources }
$$

where $C$ is the number of droplets per cubic meter, $u$ the carrier fluid velocity, $V_{s}$ the droplet settling velocity, $D$ the coefficient of molecular dispersion, $\varepsilon_{p}$ the particle turbulent dispersion coefficient. The deposition mass rate is a function of the particle concentration in the pipe, based on the following boundary condition:

$$
J_{w}=\dot{m}=\rho_{p_{w}} V_{r_{B}}+p_{t} \rho_{p_{T}} V_{r_{T}}-\left[\left(D_{B}+D_{t u r b}\right) \frac{\partial \rho_{p}}{\partial r}\right]_{w},
$$

with $\rho_{p}$ the mass concentration of droplets, $V$ the droplet velocity. Subscripts $r$ and $w$ denote radial and wall, respectively. Note that the new $p_{t} \rho_{p_{T}} V_{r_{T}}$ term accounts for the flux of drops coming from the nearest turbulent region to the wall. Drops can get high turbulent velocity from that region (around $y^{+}=20$ or 30 , here) and impact the walls without loosing their speed significantly. The $p_{t}$ parameter is present to account for the probability of such an event to occur since one part only of the drops present in that region, which have a positive radial velocity, are going to deposit. This term renders the expression slightly different from the wall flux derivation from Young \& Leeming (1997). Finally, the $-D_{\text {turb }}\left(\frac{\partial \rho_{p}}{\partial r}\right)_{w}$ term accounts for the flux of drops depositing due to turbophoresis effects.

A possible novel statistical model is proposed, based on the following approach:

- Consider the initial droplet injection as a set of point particle sources.

- Evolve the dispersion of each point-source using the assumed distribution and the moments (provided in Table 2). Adapt the given evolution for points outside the inlet center.

- Reconstruct the concentration field using the superposition of each radial distribution at a given time and position.

- Represent the wall concentration by the area of the probability density function exceeding the pipe radius $P(r>R)$

- Evaluate the mass flux with Equation 32

From the results generated in this study, such a procedure could be applied. However, this would require additional development, which is beyond the prime objective of this research work.

\section{Conclusion}

Lagrangian simulations in a vertical pipe have been performed with a modified OpenFOAM version. Simulated results agreed fairly well with experimental data and with other numerical results. A temporal and spatial study of the transient evolution of dispersion has been carried out for a wide range of droplet sizes. The final average radial position of medium size droplets converged to sub-microns droplets average radial position when looking at the space evolution. However, the final average radial position of medium size droplets converged to the large size droplets average radial position when looking at the time evolution. 
The related deposition has also been reported along with the impact velocity and a probability density function estimate of the deposition time. Such information is useful to determine how droplets disperse in the vicinity of the walls.

Turbulence is important in the deposition process. Boundary conditions of standard Eulerian models should not only be based on the wall concentration, but should also take into account the contribution of droplets issued from the turbulent layer. This has not been investigated further in this work, but has been reported elsewhere in the literature.

New insights on the droplet size dependence of both dispersion and deposition have been provided. A new statistical approach has been suggested, based on the evolution of a pre-described radial distribution and moments.

The ability to predict quickly both droplets concentration and deposition could allow a fast assessment of particles collisions, film formation or erosion related phenomena, which are important factors in safety and flow assurance evaluations.

\section{References}

Ahmadi, G., \& McLaughlin, J. B. 2008. Transport, Deposition and Removal of Fine Particles - Biomedical Applications. Pages $92-173$ of: Matijevi, Egon (ed), Medical Applications of Colloids. Springer US.

Atiya, A. F., \& M., Steve a. K. 2005. Efficient Estimation of First Passage Time Density Function for Jump-Diffusion Processes. SIAM Journal on Scientific Computing, 26(5), 1760-1775.

Berlemont, A., Desjonqueres, P., \& Gouesbet, G. 1990. Particle lagrangian simulation in turbulent flows. International Journal of Multiphase Flow, 16(1), 19-34

Bocksell, T L, \& Loth, E. 2006. Stochastic modeling of particle diffusion in a turbulent boundary layer. International Journal of Multiphase Flow, 32(10-11), 1234-1253.

Crowe, C., Sommerfeld, M., \& Tsuji, Y. 1998. Multiphase flows with droplets and particles. CRC Press.

Dallali, M., \& Armenio, V. 2015. Large eddy simulation of two-way coupling sediment transport. Advances in Water Resources, 81, 33-44.

De Marchis, M., Milici, B., Sardina, G., \& Napoli, E. 2016. Interaction between turbulent structures and particles in roughened channel. International Journal of Multiphase Flow, 78, 117-131.

Doisneau, F., Dupays, J., Murrone, A., Laurent, F., \& Massot, M. 2013. Eulerian versus Lagrangian simulation of unsteady two-way coupled coalescing two-phase flows in solid propellant combustion.

Dosio, A., Guerau de Arellano, J. V., Holtslag, A. a. M., \& Builtjes, P. J. H. 2005. Relating Eulerian and Lagrangian Statistics for the Turbulent Dispersion in the Atmospheric Convective Boundary Layer. Journal of the Atmospheric Sciences, 62(4), 1175-1191.

Einstein, A. 1905. On the movement of small particles suspended in stationary liquids required by the molecular-kinetic theory of heat.

FLUENT. 2014. User's Guide Fluent 14.0. Fluent Incorporation, USA

Gosman, A. D., \& Ioannides, E. 1983. Aspects of Computer Simulation of Liquid-Fueled Combustors. Journal of Energy, 7(6), 482-490.

Gouesbet, G., \& Berlemont, A. 1998. Eulerian and Lagrangian approaches for predicting the behaviour of discrete particles in turbulent flows. Progress in Energy and Combustion Science, 25(2), 133-159.

Govan, a H, Hewitt, G F, \& Ngan, C F. 1989. Particle motion in a turbulent pipe flow. International Journal of Multiphase Flow, 15(3), 471-481.

Graham, D. I., \& James, P. W. 1996. Turbulent dispersion of particles using eddy interaction models. International Journal of Multiphase Flow, 22(1992), 157-175

Gupta, Pankaj K, \& Pagalthivarthi, Krishnan V. 2006. A comparative study of the effect of model lift coefficients on particle trajectory. Indian Journal of Engineering $\mathcal{E}$ Materials Sciences, 13(August), 293-306.

He, C., \& Ahmadi, G. 1999. Particle deposition in a nearly developed turbulent duct with electrophoresis. Journal of Aerosol Science, 30(6).

Herpin, S. 2009. Study of the Influence of the Reynolds number on the organization of wall-bounded turbulence.

Hinze, J.O. 1975. Turbulence:. McGraw-Hill classic textbook reissue series. McGraw-Hill.

Hrenya, C M, Bolio, E J, Chakrabarti, D, \& Sinclair, J L. 1995. Comparison of low Reynolds number $k-\epsilon$ turbulence models in predicting fully developed pipe flow. Chemical Engineering Science, 50(12), 1923-1941.

Jin, C., Potts, I., \& Reeks, M. W. 2015. A simple stochastic quadrant model for the transport and deposition of particles in turbulent boundary layers. Physics of Fluids, 27(5), 1-41.

Kalitzin, Georgi, Medic, Gorazd, Iaccarino, Gianluca, \& Durbin, Paul. 2005. Near-wall behavior of RANS turbulence models and implications for wall functions. 204, 265-291.

Kallio, GA, \& Reeks, MW. 1989. A numerical simulation of particle deposition in turbulent boundary layers. International Journal of Multiphase Flow, 15(3), 433-446.

Kim, John, Moin, Parviz, \& Moser, Robert. 1987. Turbulence statistics in fully developed channel flow at low Reynolds number. Journal of Fluid Mechanics, 177(-1), 133.

Koeltzsch, K. 1998. On the relationship between the Lagrangian and Eulerian time scale. Atmospheric Environment, 33(1), 117-128.

Kou, S. G., \& Wang, H. 2003. First passage times of a jump diffusion process. Advances in Applied Probability, 35(2), 504-531.

Lai, A. C. K., \& Cheng, Y. C. 2007. Study of expiratory droplet dispersion and transport using a new Eulerian modeling approach. Atmospheric Environment, 41(35), 7473-7484.

Launder, B.E., \& Sharma, B.I. 1974. Application of the Energy-Dissipation Model of Turbulence to the Calculation of Flow Near a Spinning Disc. Letters in Heat and Mass Transfer, 1(2), 131 - 138.

Lecrivain, G., \& Hampel, U. 2012. Influence of the Lagrangian Integral Time Scale Estimation in the Near Wall Region on Particle Deposition. Journal of Fluids Engineering, 134, 074502. 
Li, A., \& Ahmadi, G. 1993. Deposition of aerosols on surfaces in a turbulent channel flow. International Journal of Engineering Science, 21(3).

Liu, B. Y. H., \& Agarwal, J. K. 1974. Experimental observation of aerosol deposition in turbulent flow. Journal of Aerosol Science, 5(2), 145-155.

Loth, E. 2000. Numerical approaches for motion of dispersed particles, droplets and bubbles. Progress in Energy and Combustion Science, 26(3), 161-223.

Marchioli, C., \& Giusti, A. 2003. Direct numerical simulation of particle wall transfer and deposition in upward turbulent pipe flow. International journal of Multiphase flow, 29, 1017-1038.

Marshall, J. S., \& Sala, K. 2013. Comparison of methods for computing the concentration field of a particulate flow. International Journal of Multiphase Flow, 56, 4-14.

Matida, E. A., Nishino, K., \& Torii, K. 2000. Statistical simulation of particle deposition on the wall from turbulent dispersed pipe flow. International Journal of Heat and Fluid Flow, 21(4), 389-402.

Menter, F.R. 1993. Zonal Two Equation k- Turbulence Models for Aerodynamic Flows. AIAA paper.

Milici, Barbara, De Marchis, Mauro, Sardina, Gaetano, \& Napoli, Enrico. 2014. Effects of roughness on particle dynamics in turbulent channel flows: a DNS analysis. Journal of Fluid Mechanics, 739, 465-478.

Pang, M. J., \& Wei, J. J. 2011. Analysis of drag and lift coefficient expressions of bubbly flow system for low to medium Reynolds number. Nuclear Engineering and Design.

Papavergos, P. G., \& Hedley, A. B. 1984. Particle Deposition Behavior from Turbulent Flows. Chemical Engineering Research and Design, 62.

Paras, S.V., \& Karabelas, A.J. 1991. Droplet entrainment and deposition in horizontal annular flow. International Journal of Multiphase Flow, 17(4), 455-468.

Picano, F., Sardina, G., \& Casciola, C. M. 2009. Spatial development of particle-laden turbulent pipe flow. Physics of Fluids, 21(2009), 093305.

Picano, Francesco, Breugem, Wim-paul, \& Brandt, Luca. 2015. Turbulent channel flow of dense suspensions of neutrally buoyant spheres. Journal of Fluid Mechanics, 764, 463-487.

Szabados, T. 2010. An elementary introduction to the Wiener process and stochastic integrals. 45.

Taylor, G. I. 1922. Diffusion by Continuous Movements. Proceedings of the London Mathematical Society, s2-20(1), 196-212.

Tian, L., \& Ahmadi, G. 2007. Particle deposition in turbulent duct flows-comparisons of different model predictions. Journal of Aerosol Science, 38(4).

Vijiapurapu, Sowjanya, \& Cui, Jie. 2010. Performance of turbulence models for flows through rough pipes. Applied Mathematical Modelling, 34(6), 1458-1466.

Vreman, A. W. 2007. Turbulence characteristics of particle-laden pipe flow. Journal of Fluid Mechanics, 584, $235-279$.

Vreman, A. W. 2015. Turbulence attenuation in particle-laden flow in smooth and rough channels. Journal of Fluid Mechanics, 773, $103-136$.

Weller, H G, \& Tabor, G. 1998. A tensorial approach to computational continuum mechanics using object-oriented techniques. Computers in Physics, 12(6), 620-631.

Young, J., \& Leeming, A. 1997. A theory of particle deposition in turbulent pipe flow. Journal of Fluid Mechanics.

Zhang, Z., \& Chen, Q. 2007. Comparison of the Eulerian and Lagrangian methods for predicting particle transport in enclosed spaces. Atmospheric Environment, 41(25), 5236-5248. 